\title{
Une expérience de lutte génétique contre Culex pipiens fatigans Wied., 1828
}

\author{
par H. LAVEN
}

Institut für Genetik, Johannes Gutenberg Universität - Mainz, Allemagne

\begin{abstract}
Résumé
Le croisement entre les membres de populations allopatriques du complexe de Culex pipiens peut donner lieu à quatre résultats différents. La majorité des populations produit une descendance normale dans les croisements réciproques alors que certains autres donnent des descendants dans une direction et que les embryons n'éclosent pas dans la direction opposée. D'autres croisements sont inféconds dans les deux directions. Cette absence de descendance est due à une incompatibilité cytoplasmique qui est cytoplasmiquement héréditaire. Elle demeure constante dans la lignée femelle pendant un nombre indéfini de générations. Le spermatozoïde est bloqué dans un croisement incompatible avant qu'il ne fusionne avec le noyau ovulaire haploïde et, si des embryons se développent, ils le font à partir d'un ovule haploïde et meurent avant l'éclosion.

On connaît vingt types différents de croisement dans le complexe de Culex pipiens et il est possible de préciser quelle est la souche du complexe, ou parfois les souches, qui sont incompatibles avec une certaine population de Culex pipiens d'un point donné du globe. Il est possible d'introduire des caractères géniques désirables dans une souche incompatible sans changer l'incompatibilité cytoplasmique et l'on peut adapter ainsi des souches de régions tempérées à des environnements tropicaux.

Il serait souhaitable d'effectuer des lâchers de mâles incompatibles dans une population naturelle dans le cadre de la lutte contre Culex pipiens. Des expériences effectuées dans des cages montrent que les femelles sont incapables de faire la discrimination entre les mâles normaux et les mâles incompatibles; la compétitivité des
\end{abstract}


mâles dépend en grande partie des conditions de l'alimentation au cours du développement larvaire. Des populations d'élevage, présentant un taux initial d'un mâle incompatible pour un mâle normal, ont été éradiquées en trois ou quatre générations.

L'Organisation Mondiale de la Santé a cautionné une expérience pilote effectuée avec la collaboration de l'Unité de Recherches sur les Filarioses à Rangoon. On utilisa une souche de Culex pipiens fatigans ayant le cytoplasme d'une souche de Paris et le génome d'une souche de Fresno (Californie) considérée comme étant mieux adaptée aux conditions écologiques de la Birmanie que la souche pure de Paris. D'août à octobre 1966, on testa l'incompatibilité des mâles avec des femelles provenant de vingt-cinq populations naturelles de Rangoon et de ses environs. Ces femelles déposèrent au total 1.472 pontes où l'on décomptait 130.455 œufs. 180 larves seulement arrivèrent à l'éclosion $(0,14 \%)$.

Le travail sur le terrain fut effectué à Okpo, village situé à $25 \mathrm{~km}$ environ au nord de Rangoon, entouré de rizières sèches pendant l'hiver. On estima que la densité de la population de moustiques variait d'un minimum de 2.000 mâles et 2.000 femelles jusqu'à 10.000 mâles et 10.000 femelles suivant les jours, cette variation dépendant surtout des fluctuations du nombre et de l'étendue des gîtes larvaires.

Des mâles incompatibles furent lâchés entre février et mai 1967. On lâcha tous les jours 5.000 mâles incompatibles du 16 mars au 6 mai. En raison du nombre initial bas de lâchers, le pourcentage de pontes non écloses provenant de croisements incompatibles demeurait bas (semaines $1-4 ; 4,4-11,6 \%$ ). Peu après le lâcher d'un nombre optimal de 5.000 mâles incompatibles par jour (flèche dans la fig. 1), le pourcentage de pontes inviables augmenta jusqu'à une moyenne de 19,4\% (cinquième semaine, 21 au 27 mars) en dépit d'un accroissement presque décuplé de la taille de la population au cours des quatre premières semaines (70 pontes par jour pendant la première semaine, 625 pontes par jour durant la quatrième semaine). On constata au cours des sept jours suivants (28 mars au 3 avril) un léger acoroissement jusqu’à $24,8 \%$; durant la semaine suivante ( 4 au 10 avril), on atteignait $30,7 \%$. Le pourcentage des pontes inviables s'accrut alors plus rapidement. Il atteignait $39,0 \%$ du 11 au 17 avril (huitième semaine) et 70,4\% du 25 avril au $1^{\text {er }}$ mai (dixième semaine). Une nouvelle poussée de production élevée dans les gîtes larvaires, débutant à la dixième semaine, fut de toute évidence rendue inefficace par le haut pourcentage de pontes sans éclosion. Du 2 au 8 mai (onzième semaine), le pourcentage atteignit $85,5 \%$ et l'on obtenait enfin $100 \%$ de pontes inviables les 9 et 10 mai. La production de nouvelles larves était donc arrêtée et aucun adulte ne devait éclore après dix jours environ. La mousson débuta malheureusement le 11 mai et l'expérience dut être interrompue. 
Les résultats de cette expérience montrent que le facteur d'incompatibilité est capable d'agir dans la nature et qu'après le lâcher d'un nombre adéquat de mâles incompatibles, on peut éradiquer une population de $C$. fatigans en trois mois environ ou en cinq ou six générations.

\section{Summary}

Crossing between members of allopatric populations of the Culex pipiens complex can produce four different results. Most populations will produce normal offspring in reciprocal crosses, while some give offspring in one direction and embryos which will not hatch in the opposite direction. Other crosses are infertile in both directions. This lack of offspring is due to cytoplasmic incompatibility, which is inherited cytoplasmically. It remains constant for indefinite numbers of generations in the female line. In an incompatible cross the sperm is blocked before it can fuse with the haploid egg nucleus, and if the embryos develop they do so from the haploid egg nucleus and die before hatching.

Twenty different crossing types are known in the Culex pipiens complex, and it is possible to specify at least one, sometimes several, strains of the complex which are incompatible with a certain population of Culex pipiens anywhere in the world. Desirable genic traits can be introduced into an incompatible strain without changing the cytoplasmic incompatibility, and so strains from temperate regions can be adapted to tropical environments.

Release of incompatible males into a natural population should be suitable for the control of Culex pipiens. Cage experiments show that females cannot discriminate between normal and incompatible males, and the competitiveness of males depends largely on nutritional conditions during larval development. Cage populations with an initial ratio of incompatible and normal males of 1: 1 have been eradicated after three or four generations.

The World Health Organization sponsored a pilot experiment at the Filariasis Research Unit at Rangoon. A strain of Culex pipiens fatigans with the cytoplasm from a strain from Paris and the genome from a strain from Fresno, California, considered to be better adapted to ecological conditions in Burma than the pure Paris strain, was used. During August to October 1966, males were tested for incompatibility with females from twenty-five natural populations in Rangoon and its surroundings. These females laid a total of 1,472 rafts with 130,445 eggs. Only 180 larvae hatched ( 0.14 per cent). 
The field work was carried out at Okpo, a village about 10 miles north of Rangoon, surrounded by completely dry rice fields during winter. The size of the mosquito population was estimated to vary from a minimum of 2,000 males and 2,000 females to as many as 10,000 males and 10,000 females on a given day, depending mostly on fluctuations in the number and extent of breeding places.

Incompatible males were released between February and May 1967. From March 16 until May 6, 5,000 incompatible males were released daily. Because of the initially small number released the percentage of non hatching rafts from incompatible crosses remained low (week 1-4, 4.3-11.6 per cent). Soon after the release of the optimum number of 5,000 incompatible malesi day (arrow in Fig. 1) the percentage of inviable rafts increased to an average of 19.4 per cent (fifth week, March 21-27) in spite of an almost ten-fold increase in the size of the population during the first 4 weeks (seventy rafts/day during the first week, $625 \mathrm{rafts} /$ day during the fourth week). During the next seven days (March 28 to April 3) a slight increase to 24.8 per cent occurred; during the following week (April 4-10) 30.7 per cent was observed. From then on the percentage of inviable rafts increased more rapidly. It reached 39.0 per cent during April 1117 (week 8) and 70.4 per cent during April 25 to May 1 (week 10). A new wave of higher production in the breeding places, beginning in week 10, was obviously cut off by the high percentage of non-hatching rafts. During May 2-8 (week 11) the percentage increased to an average of 85.5 per cent and finally, on May 9 and 10,100 per cent inviable rafts were obtained. The production of new larvae was therefore stopped and no more adults were expected to emerge after about 10 days. Unfortunately, the monsoons started on May 11 and the experiment had to stop.

The results of this experiment show that the incompatibility principle can operate in nature and that, after the release of an adequate number of incompatible males, a C.p. fatigans population was eradicated in about 3 months or five or six generations.

\section{Introduction}

L'expérience sur le terrain que je tente de décrire ici avec quelques détails est connue sous le nom d'expérience de Birmanie ou de Okpo ; elle est basée sur une vingtaine d'années de recherches et d'expériences concernant l'hérédité formelle et cytoplasmique des membres du complexe Culex pipiens. Finalement, en 1963, je proposais ce sujet à l'O.M.S. pour une étude sur le terrain, mais c'est seulement en 1967, que cette expérience sur le terrain devint effective, malgré des difficultés de toutes sortes. Une communication préliminaire a déjà été publiée (Laven, 1967 c). 
Le principe de cette expérience est basé sur l'incompatibilité cytoplasmique (Laven 1953, 1957 b, 1959). Ce phénomène avait déjà été observé par Marschall (1938) et Roubaud (1941) mais une analyse précise ne fut possible qu'après la production et l'isolement de mutations visibles, utilisées comme marqueurs de chromosomes (Laven, 1955, 1956, 1957 a, Kitzmiller, 1958).

\section{Incompatibilité cytoplasmique}

$\mathrm{Si}$ deux populations allopatriques du complexe Culex pipiens sont croisées de deux façons différentes $\left(q \mathrm{~A} \times \delta^{*} \mathrm{~B}\right.$ et $\left.q \mathrm{~B} \times \sigma^{\star} \mathrm{A}\right)$ le résultat peut correspondre à l'une des quatre possibilités suivantes (Fig. 1):

\begin{tabular}{c|c|c|} 
& $\mathrm{A} \times \mathrm{O}^{\mathrm{T}} \mathrm{B}$ & $\mathrm{B} \times$ 丁 $^{\mathrm{A}} \mathrm{A}$ \\
\hline 1 & + & + \\
\hline 2 & + & - \\
\hline 3 & - & + \\
\hline 4 & - & - \\
\hline
\end{tabular}

FIG. 1. - Les quatre résultats possibles des croisements réciproques entre une population $\mathrm{A}$ et une population B de Culex pipiens (voir le texte pour d'autres explications)

$1^{\circ}$ Une descendance normale dans les deux sens.

$2^{\circ}$ Une descendance normale dans le sens $\left(+\mathrm{A} \times \delta^{\top} \mathrm{B}\right)$, mais pas de descendance dans le sens $\left(q \mathrm{~B} \times \delta^{*} \mathrm{~A}\right)$.

$3^{\circ}$ Une descendance normale dans le sens inverse $\left(q \mathrm{~B} \times \sigma^{*} \mathrm{~A}\right)$ et aucun résultat dans l'autre sens $\left(\stackrel{+}{A} \times \delta^{\wedge} \mathrm{B}\right)$.

$4^{\circ}$ Aucune descendance dans les deux sens.

Le premier cas correspond à un croisement normal comme cela se passe entre des populations différentes d'une même espèce. Les deuxième et troisième cas démontrent une incompatibilité unidirectionnelle et le dernier cas une incompatibilité bidirectionnelle.

Si une même population est croisée avec une série de populations d'origines géographiques différentes, on obtient un certain modèle de croisement qui a été appelé type de croisement. Une population peut être normalement compatible avec une autre population, montrer une incompatibilité dans un sens avec une seconde population, dans le sens opposé avec une troisième et avec une quatrième, produire à nouveau une descendance dans les deux sens, et ainsi de suite. En testant une centaine de populations différentes à travers le monde, excepté l'Australie et l'Amérique du Sud, nous avons trouvé environ vingt types de croisements différents, dont cinq seulement en Europe. 
Le type de croisement est une caractéristique stable d'une population donnée au moins dans les souches de laboratoire. Notre plus ancienne souche est autogène, provenant, vers 1935, d'une capture dans un hôpital d'enfants de Hambourg. Depuis ce temps, elle est conservée en vie presque exclusivement sans repas de sang. Nous avons testé cette souche pour la première fois en 1947, avec différentes autres souches européennes et un certain type de croisement a été établi. Depuis, de temps en temps, nous contrôlons le type de croisement de la souche qui se révèle identique depuis 22 ans. D'autres souches sont en observation depuis la même date et ne présentent aucun changement de type. $\mathrm{Si}$, par hasard, des changements se produisent, ils doivent être très rares. Ces remarques sur la stabilité du type de croisement devraient sans aucun doute être valables pour toute souche incompatible, sélectionnée à partir d'expériences sur le terrain, que cette souche dérive d'une population naturelle, ou d'une souche composite formée du cytoplasme d'une population et des chromosomes d'une autre.

L'incompatibilité, c'est-à-dire l'absence de descendance, présente les caractéristiques suivantes. La copulation est normale, les œufs sont fécondés. Les mâles incompatibles ont des spermatozoïdes parfaitement viables, comme on a pu le démontrer dans des croisements de tels mâles avec leurs sœurs. La pénétration du spermatozoïde stimule la méïose du noyau de l'œuf aussi bien dans un croisement normal que dans un croisement incompatible, mais après la division méiotique complète, l'évolution dans un croisement normal et dans un croisement incompatible est différente. Dans le croisement normal le noyau du spermatozoïde qui a un jeu de chromosomes haploïdes fusionne avec le noyau de l'ovule qui contient le complément haploïde des chromosomes. Dans un croisement incompatible, le spermatozoïde est bloqué en caryogamie et se désintègre. Ainsi le spermatozoïde ne peut contribuer à aucun développement ultérieur de l'embryon. Des expériences génétiques faisant intervenir la transmission de mutation par le spermatozoïde ont démontré ces faits. On n'a jamais pu observer le développement d'un embryon après un croisement incompatible. On a suivi la pénétration et la désintégration du spermatozoïde par traceurs radio-actifs.

Dans un croisement incompatible, le développement embryonnaire débute dans un pourcentage d'œufs très élevé, quelquefois dans presque tous les œufs. Ce développement doit être basé sur le lot haploïde de chromosomes, que ce lot haploïde de gènes possède l'information suffisante au développement embryonnaire, ou que les gènes ne soient pas actifs. L'information pourrait provenir de l'A.R.N. contenu dans le cytoplasme de l'ovule. Dans chaque cas, le développement peut aller très loin, presque jusqu'aux derniers stades précédant l'éclosion. Mais, comparés aux embryons normaux, ces embryons ont une apparence anormale et irrégulière. Ces remarques présentent un certain intérêt pour des expériences sur le terrain. Par une étude des pontes, on peut aisément différencier une ponte non fécondée présentant un aspect blanchâtre sans trace de développement, de pontes provenant de croisements incompatibles dont le pourcentage de développement embryonnaire anormal est variable et de pontes parvenant normalement à l'éclosion.

Il faut noter que dans les croisements incompatibles, moins de 0,5\% des œufs éclosent et qu'une partie des larves donnera des femelles adultes. Il y a eu dans ces 
cas une diploïdisation du noyau de l'ovule, et ces femelles sont parfaitement normales, viables et fertiles.

Pour résumer les caractéristiques d'un croisement incompatible, nous pouvons dire que la copulation et l'insémination ont lieu, que le spermatozoïde est bloqué après son entrée dans l'ovule, qu'il y a un pourcentage élevé de développement embryonnaire, mais que la plupart des embryons meurent avant l'éclosion, et que moins de 0,5\% peuvent devenir des femelles parthénogénétiques.

Pourquoi avons-nous appe:é ce phénomène incompatibilité cytoplasmique? Les deux souches allemandes, Hambourg (Ha, Allemagne du Nord) et Oggelshausen (Og, Allemagne du Sud) montrent une incompatibilité unidirectionnelle, c'est-à-dire, que les femelles Ha peuvent produire une descendance normale avec des mâles Og, mais le croisement inverse des femelles $\mathrm{Og}$ avec des mâles $\mathrm{Ha}$ est incompatible. Très tôt dans nos recherches sur l'incompatibilité, nous nous sommes demandé quel serait le type de croisement des hybrides provenant de femelles $\mathrm{Ha}$ et de mâles $\mathrm{Og}$. Les tests de croisement de la première génération d'hybrides révélèrent que ceux-ci étaient exactement identiques à la souche $\mathrm{Ha}$ en dépit de la composition chromosomique, moitié chromosomes $\mathrm{Ha}$ et moitié chromosome $\mathrm{Og}$. Se comportant comme des femelles $\mathrm{Ha}$, les femelles hybrides de $\mathrm{F} 1$ peuvent à nouveau se croiser en retour avec les mâles Og. Les hybrides F2 devraient donc avoir théoriquement en moyenne les trois quarts de chromosomes de $\mathrm{Og}$ et seulement un quart de Ha. Nous avons répété ces croisements en retour sur 52 générations (fig. 2), pendant plus de cinq ans. Il n'est pas douteux que nous ayions remplacé dans cette longue série de générations tous les chromosomes de la souche originale par les chromosomes de la souche Og. On testait les hybrides, mâles aussi bien que femelles, au début de chaque génération, puis, plus tard, occasionnellement dans une génération ou une autre. Les hybrides mâles, ayant de plus en plus de chromosomes $\mathrm{Og}$, auraient dû être modifiés en ce qui concerne la possibilité de croisement avec les femelles $\mathrm{Og}$ (fig. 3, colonne de gauche) et les hybrides femelles auraient dû devenir incompatibles avec les mâles Ha (fig. 3, colonne de droite). Mais il ne se produisit rien de cette sorte. Les hybrides de chaque génération jusqu'à la $52^{\circ}$ se comportèrent exactement comme les animaux de la souche Ha. Le remplacement des chromosomes ne change donc pas le type de croisement. Nous concluons que la réaction entre l'œuf et le spermatozoïde dans un croisement incompatible est déterminée par des facteurs cytoplasmiques extra-chromosomiques et nous appelons ce phénomène incompatibilité cytoplasmique. On ne connaît pas encore la nature de la réaction entre le cytoplasme de l'œuf et le spermatozoïde. Mc Clelland (1967) a expliqué que le phénomène était dû à une ségrégation polarisée sans donner aucune preuve de son existence chez Culex. Les nouvelles méthodes génétiques nous ont fourni de nouvelles preuves de la possibilité de remplacement total des chromosomes, confirmant la supposition d'une ségrégation polarisée, et corroborant l'interprétation de l'hérédité cytoplasmique dans le type de croisement. Nous verrons par la suite que la possibilité du remplacement des chromosomes sans changement du type de croisement a quelque importance pour le développement des 

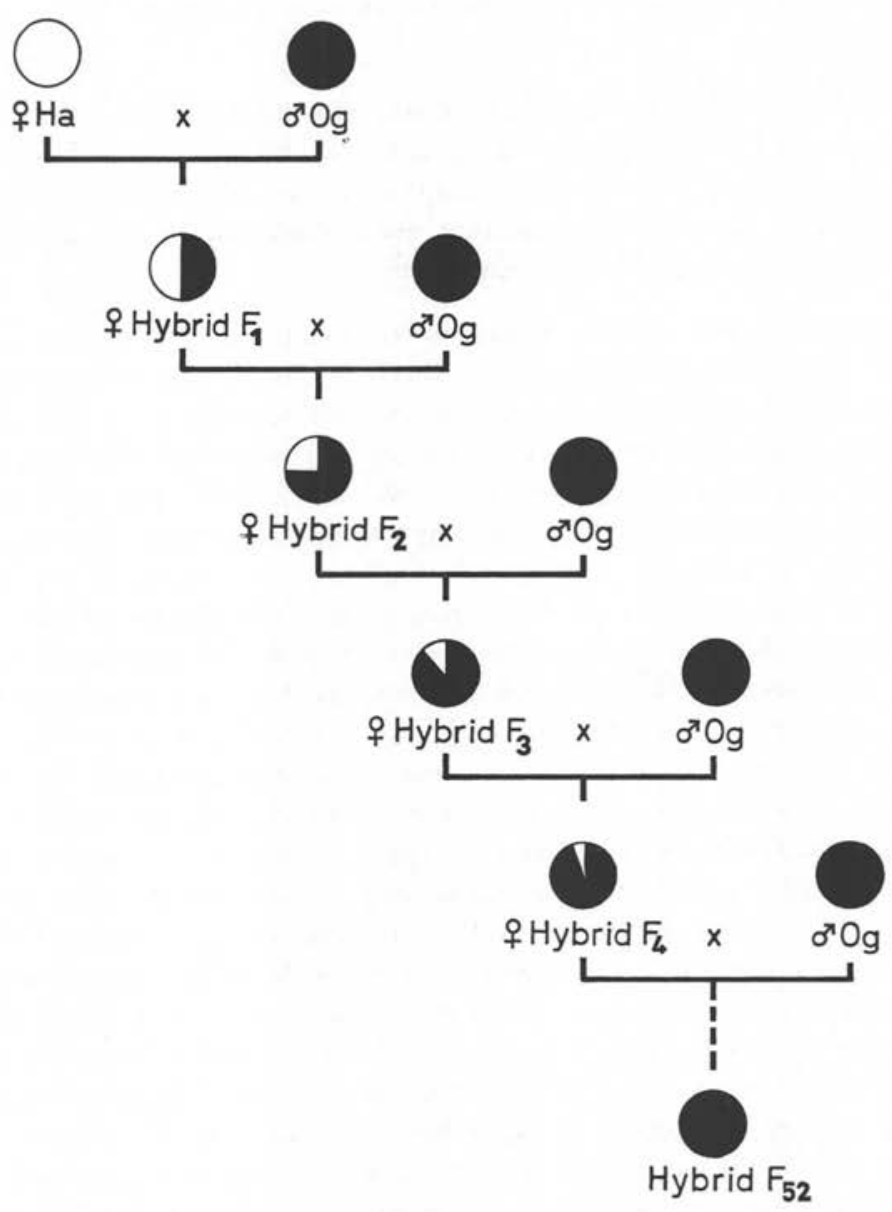

Fig. 2. - Procédé de croisements en retour continus d'hybrides provenant d'un croisement initial entre une femelle de la souche Hambourg (Ha) et un mâle de la souche Oggelshausen $(\mathrm{Og})$. Les chromosomes des deux souches sont indiqués par les parties blanches et noires des cercles. L'hybride femelle de la génération F1 a un lot chromosomique composé pour moitié des chromosomes de $\mathrm{Ha}$, l'autre moitié provenant de $\mathrm{Og}$. Tous les hybrides F2 n'ont dans l'ensemble qu'un quart de chromosomes $\mathrm{Ha}$ et trois quarts de chromosomes $\mathrm{Og}$. Les chromosomes $\mathrm{Ha}$ diminuent à chaque génération

souches incompatibles adaptées aux conditions de l'environnement, dans une zone où on a l'intention d'entreprendre une expérience sur le terrain.

Comme nous l'avons déjà dit, l'incompatibilité cytoplasmique conduit à une absence presque totale de descendants. Il est évident que l'on pourrait utiliser ce mécanisme comme méthode de contrôle. C'est un mécanisme naturel qui cause des 


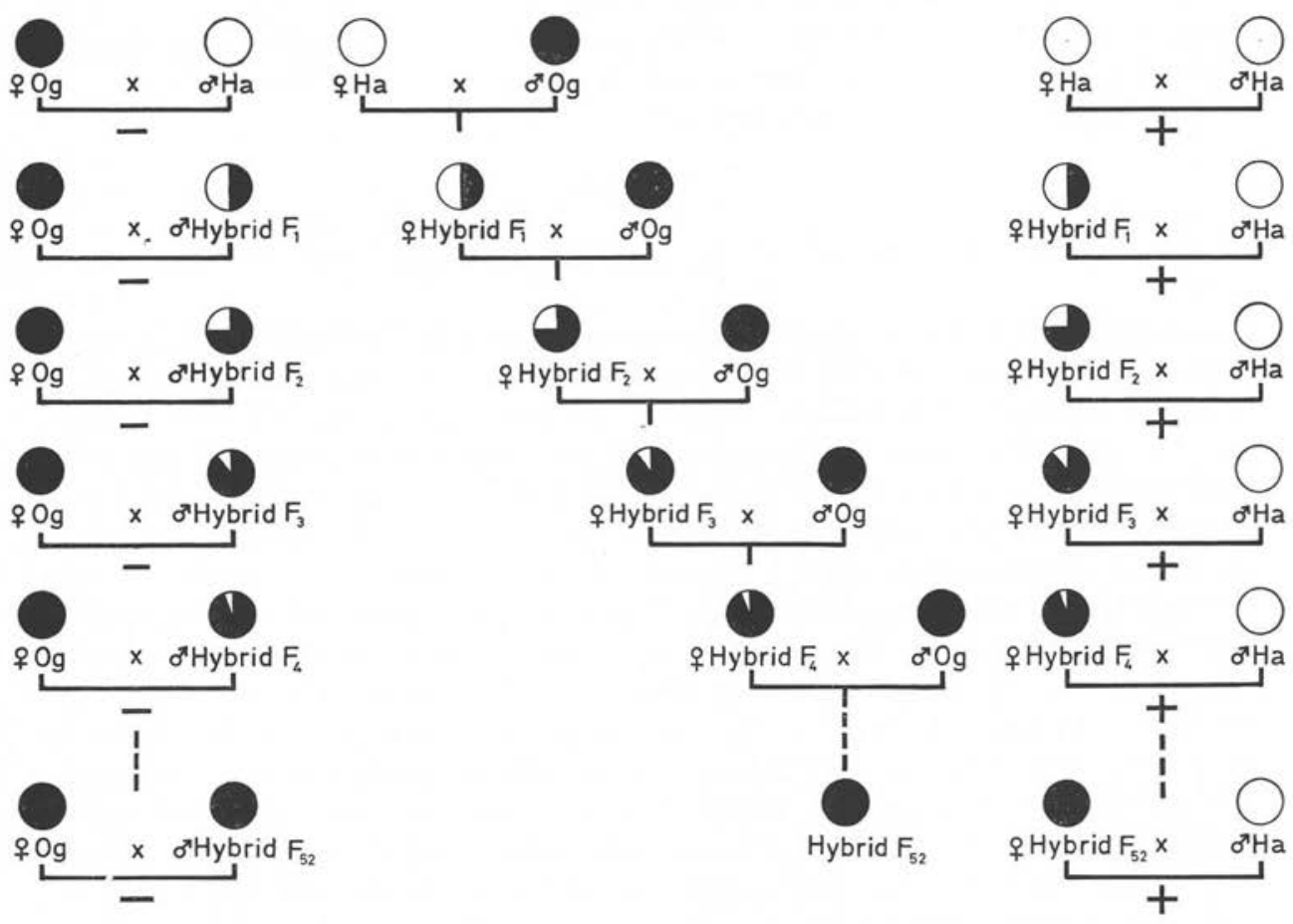

FIG. 3. - Croisement en retour d'hybrides femelles $\mathrm{Ha} \times \mathrm{Og}$ avec des mâles $\mathrm{Og}$ (colonne centrale comme dans la figure 2). Expérience de croisement d'hybrides mâles avec des femelles de la souche paternelle Og (colonne de gauche): incompatibilité constante. Expérience de croisement d'hybrides femelles avec des mâles de la souche maternelle (colonne de droite): compatibilité constante

dommages dont les conséquences possibles sont très importantes, à savoir l'empêchement total de la reproduction. Il apparaît dans une seule et même espèce et il est donc en rapport étroit avec tout effet nuisible sur la vigueur ou l'adaptabilité du porteur d'un certain type de croisement. Il n'y a de plus aucune différence de comportement copulatoire entre les divers types de croisement, donc pas de barrières entre des populations différentes. Une série d'expériences en cage nous a montré que ces observations sont exactes et qu'un lâcher de mâles incompatibles dans la proportion de 1 pour 1 parmi une population stable en cage pouvait conduire à l'éradication totale de cette population en quelques générations. En me basant sur une longue expérience des aspects variables de la biologie et de la génétique du Culex, je fis en 1963 une proposition pour un essai de contrôle des populations de Culex sur le terrain, par l'usage de mâles incompatibles, comme je l'ai mentionné dans l'introduction. Trois ans après, les derniers préparatifs, pour cette expérience de terrain en Birmanie, étant terminées, Barr (1966) publia un court papier sur l'utilisation de l'incompatibilité cytoplasmique comme moyen de contrôle du Culex pipiens dans lequel il émettait des doutes 
sérieux sur l'utilité de ces méthodes. Mais l'expérience réalisée sur le terrain en 1967 a montré qu'on pouvait obtenir une éradication en utilisant le mécanisme de l'incompatibilité cytoplasmique bien que certains puissent encore en douter. Mais je reviendrai sur ces critiques à la fin de mon article.

\section{Recherches préliminaires à une expérience sur le terrain}

Après avoir établi les principales données concernant l'incompatibilité cytoplasmique de Culex pipiens et après l'accord de l'O.M.S. pour une expérience sur le terrain, le travail préliminaire qui occupa les années 1965-1966 passa par différentes étapes. Avant tout il fallut trouver un lieu convenable pour une telle expérience et être d'accord sur le lieu. Après l'inspection d'une certaine région à Ceylan, de différentes autres en Inde, ainsi que des environs de Rangoon en Birmanie, nous décidâmes que les villages près de Rangoon convenaient le mieux pour un essai initial sur le terrain. Ils présentaient la caractéristique la plus importante pour une telle expérience : ils étaient assez isolés, au moins durant la saison sèche. Finalement le petit village d'Okpo à $25 \mathrm{~km}$ au nord, nord-ouest du centre de Rangoon fut choisi comme village expérimental (Fig. 4). Au Nord, à l'Ouest et au Sud, le village est isolé par de grandes étendues de rizières. A l'Est, l'isolement n'est pas aussi bon. Le village voisin, Bonshegon, n'est situé qu'à $320 \mathrm{~m}$, séparé d'Okpo par des rizières et des buissons épars. Au cours de la saison sèche, nous avons observé qu'il n'y avait à Okpo aucun apport de moustiques provenant du village voisin Bonshegon, Okpo ressemblant ainsi à une véritable île dont la population de moustiques est bien isolée. Le petit village de Thadugan à $1 \mathrm{~km}$ au nord-nord-est d'Okpo fut choisi comme village témoin, aucun lâcher n'y fut fait.

Okpo s'étend sur environ $450 \mathrm{~m}$ d'Est en Ouest (fig. 5) et $200 \mathrm{~m}$ du Nord au Sud. Le village contient 145 maisons habitées numérotées de 208 à 355, et un petit monastère à l'extrémité Est. Au moment de la reconnaissance initiale des lieux et de l'expérience actuelle de lâcher (1966-1967) la population a été évaluée à 700 individus environ. Les maisons sont pour la plupart construites sur pilotis, le plancher étant à un mètre du sol. Elles sont faites en bambou ou en bois, couvertes d'un toit de chaume ou de tôle ondulée (Fig. 6). La plupart ne possède qu'une pièce avec une ou plusieurs dépendances et une petite cuisine.

Tous les villages autour de Rangoon sont abrités sous une épaisse frondaison d'arbres. Seule la partie sud-est d'Okpo n'est pas aussi protégée, à cause d'une extension récente du village. Un petit ruisseau traverse le village d'Est en Ouest mais il ne contient de l'eau que pendant la saison des pluies. Il n'y a pas de puits dans le village. L'eau de boisson et l'eau ménagère sont tirées de plusieurs mares artificielles réparties autour du village. Ce sont les seuls points d'eau fonctionnels durant la saison sèche mais ils ne correspondent pas à des gîtes de Culex p. fatigans. La ponte de cette espèce est limitée aux récipients laissés autour des maisons: cruches en terre cuite de toutes formes et de toutes tailles, bassins, cylindres en métal, petits pots et bols.

Ayant trouvé que le village d'Okpo convenait à une telle expérience sur le terrain, 


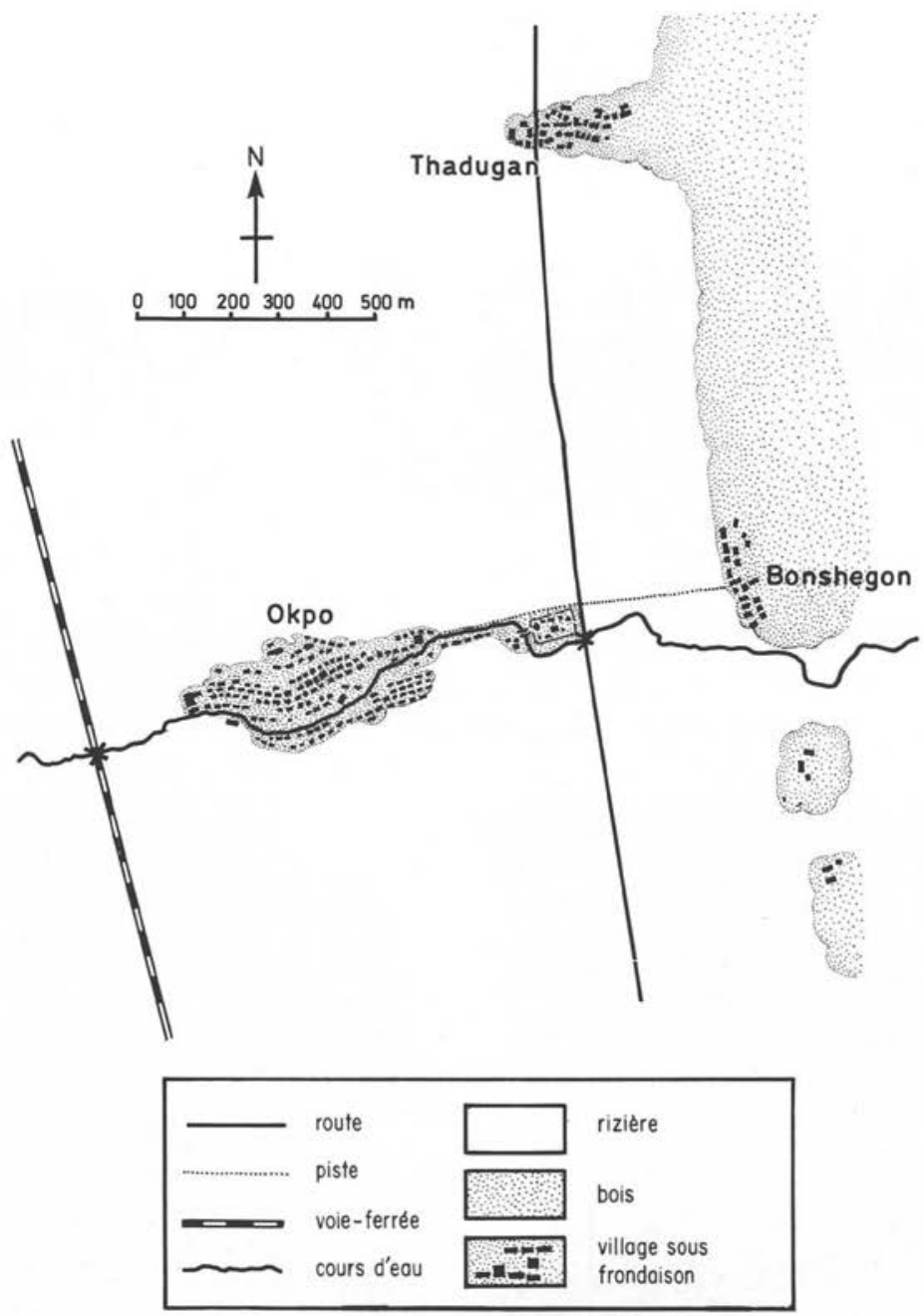

Fig. 4. - Carte du village birman d'Okpo et de ses environs

nous abordâmes la seconde étape du travail préparatoire: la sélection d'une souche incompatible, c'est-à-dire une souche dont les mâles sont incompatibles avec les femelles locales d'Okpo. Une colonie de Culex p. fatigans de Rangoon fut élevée dans notre laboratoire de Mayence et fut testée avec toute une série de souches provenant d'autres régions (Fig. 7). Quatre des neuf souches étaient incompatibles avec la souche de Rangoon, lorsqu'on croisait femelles de Rangoon et mâles d'autres souches, trois de ces souches testées présentaient une incompatibilité bidirectionnelle, la $4^{\circ}$ souche (Fno) une incompatibilité unidirectionnelle. 


\section{Okpo}

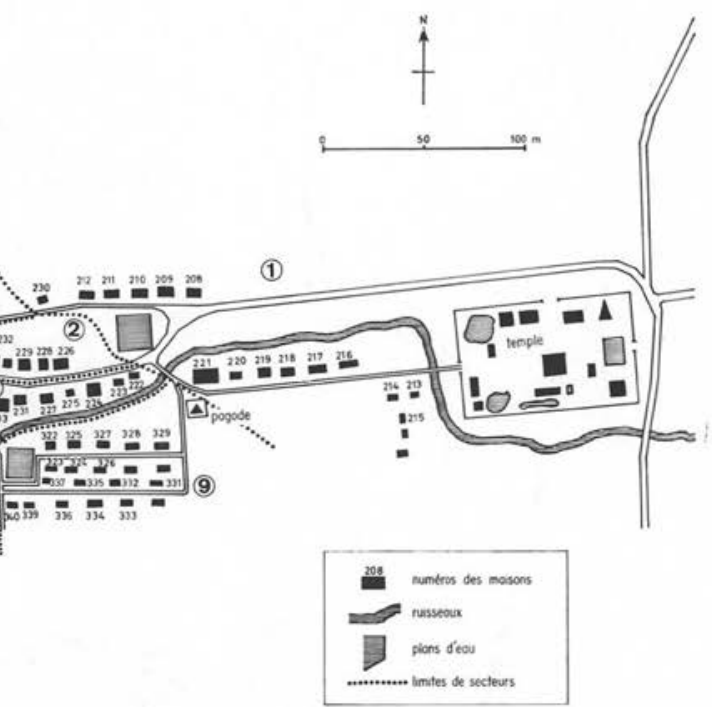

Fig. 5. - Carte du village d'Okpo montrant les routes, les ruisseaux et les marais

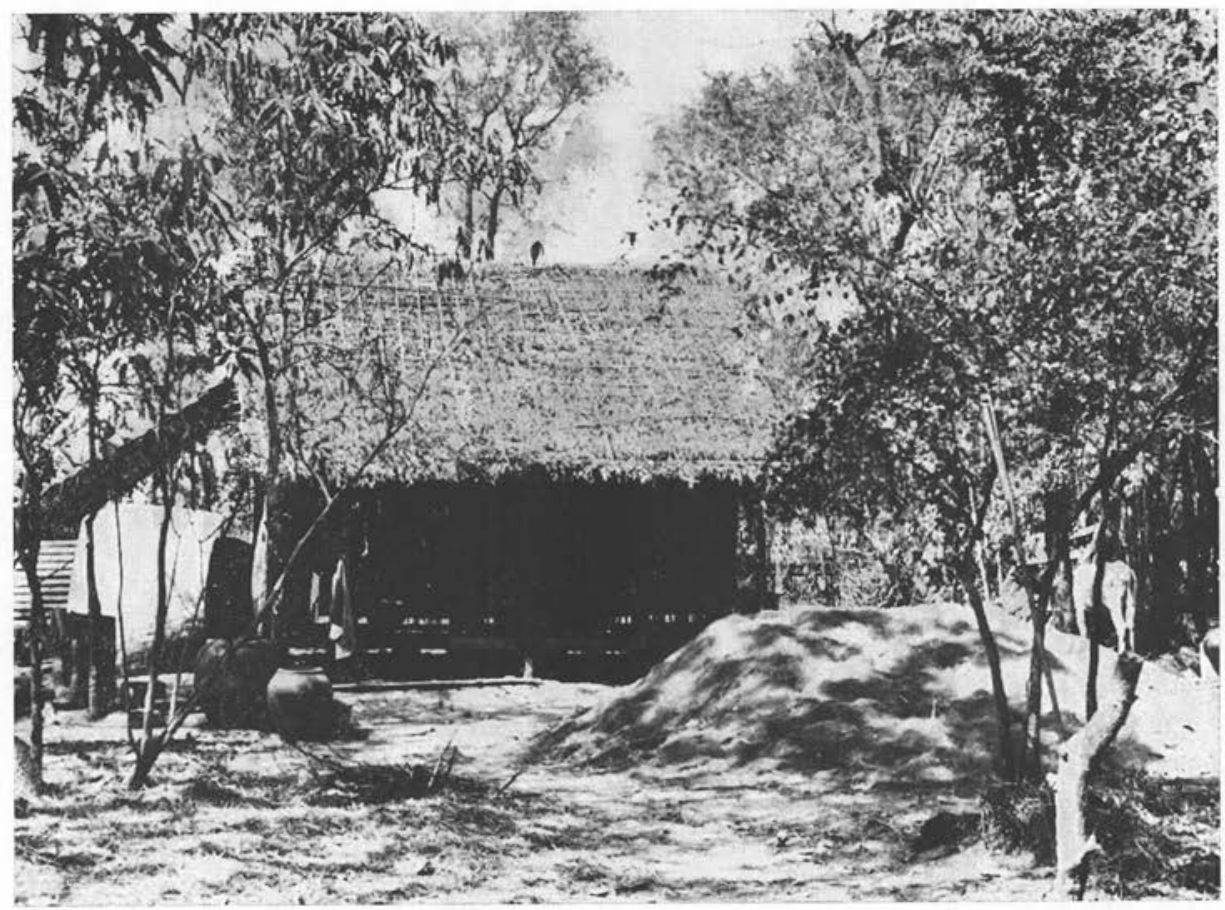

Fig. 6. - Maison typique du village d'Okpo avec des jarres en terre au premier plan 
La souche Paris $(\mathrm{Pa})$ fut sélectionnée comme étant la meilleure grâce à sa productivité élevée et à sa vigueur dans les conditions de laboratoire. Mais la souche de Paris étant originaire d'une région à climat tempéré, nous hésitions à nous en servir à l'état pur. Comme le montre le paragraphe précédent, il est possible de remplacer les chromosomes d'une souche par les chromosomes d'une autre souche sans changer le type de croisement. Nous avons utilisé ce principe pour élever une souche qui était sensée présenter une meilleure adaptation à l'environnement tropical de Birmanie que la souche Paris à l'état pur. Nous avons introduit par des croisements appropriés, les chromo-
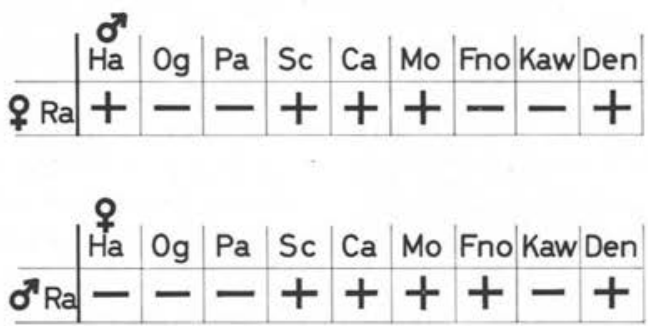

Fig. 7. - Résultats des croisements entre des femelles et des mâles de la souche de Culex $p$. fatigans de Rangoon, Birmanie $(=\mathrm{Ra})$ et de souches de pipiens ou de fatigans d'autres régions. $\mathrm{Ha}=$ Hambourg, nord de l'Allemagne ; $\mathrm{Og}=$ Oggelshausen, sud de l'Allemagne $; \mathrm{Pa}=$ Paris, France $; \mathrm{Sc}=$ Scaurie, Italie $; \mathrm{Ca}=$ Le Caire, Egypte; Mo = Montgomery, Alabama, U.S.A. ; Fno $=$ Fresno, Californie, U.S.A. ; Kaw $=$ Kawasaki, Japon $;$ Den $=$ Denken, Japon. $+=$ croisement compatible avec descendance normale, $-=$ croisement incompatbile sans descendance

somes soit de la souche Fresno (Californie), soit de la souche Freetown (Sierra Leone, Afrique). Nous ne sommes pas sûrs de l'identité de la souche réellement utilisée pour cette technique, car à cause de l'insouciance d'un technicien les deux souches avaient été soit mélangées, soit substituées. Néanmoins, nous avons introduit d'autres chromosomes dans la souche Paris donc d'autres gènes donnant une meilleure adaptation aux conditions tropicales. Les croisements en retour furent effectués pendant 8 générations. Une expérience récente utilisant la même technique et des souches similaires a-montré qu'après trois générations de croisements en retour, une telle souche composite présentait une capacité de reproductivité normale sous les conditions tropicales, fait-en contradiction avec plusieurs essais tentés dans différents laboratoires de l'Asie du Sud-Est, afin d'établir des cultures de souches pures en provenance d'Europe. En même temps qu'on cultivait la souche incompatible pour la Birmanie, une autre souche était préparée pour les populations de Culex de l'Inde, en utilisant la souche de Londres comme donneur de l'incompatibilité et la souche Freetown comme donneur de chromosomes. Les deux souches appelées $D_{1}$ et $D_{2}$ montrèrent la même capacité de reproduction dans les conditions tropicales et la même compétitivité envers les populations locales de Birmanie 
et de l'Inde. Malheureusement, les deux souches ne résistèrent pas longtemps parce qu'à un moment critique les conditions nécessaires n'étaient pas remplies à Rangoon ni en Inde, pour maintenir les souches à l'abri des contaminations et pour prévenir le danger possible de fuite du laboratoire et l'établissement dans la région. Aussi je dus conseiller dans les deux cas de détruire les souches.

Les observations sur une série de souches égyptiennes dont l'origine géographique s'étendait d'Alexandrie au Nord à Assouan au Sud (Laven 1969) ont montré que des souches ayant des types de croisements différents pouvaient exister côte à côte dans des lieux sans barrière géographique évidente. Une telle situation apparaît probablement grâce à l'introduction par voie maritime ou aérienne d'une souche étrangère. Cela aurait pu arriver également à Rangoon. Donc il était important de savoir si la ville de Rangoon et ses alentours étaient peuplés par un seul type de Culex $p$. fatigans réagissant de façon uniforme avec la souche incompatible. Au moment où les tests de croisements furent faits entre des échantillons de la population locale et de la souche $D_{1}$, nous avions èncore l'intention d'étendre l'expérience à la ville, si le premier essai réussissait. C'est pourquoi des échantillons furent pris en vingt-cinq points différents couvrant toute la région, plus ou moins espacés et répartis depuis la commune et les villages jusqu'au Nord. Les stations de capture sont indiquées par des points, sur la carte (Fig. 8), et le nom de ces lieux apparaît dans la seconde colonne du tableau 1.

Pour les essais, on utilise deux sortes d'échantillons de population. On a prélevé les nymphes dans des gîtes larvaires de haute densité pour les essais $n^{\circ s} 3,6$ et 9 . Au laboratoire, nous avons sélectionné et isolé les nymphes femelles. Nous avons mis en vage avec des mâles $\mathrm{D}_{1}$ les femelles nouvellement écloses et nous avons étudié, dans le résultat des pontes, les caractéristiques de l'incompatibilité, c'est-à-dire l'embryogénèse et la mort des embryons avec à l'exception de très faibles pourcentages de femelles parthénogénétiques. Nous avons fait tous les autres tests à partir de pontes prélevées dans la nature. On élevait les larves au laboratoire et les femelles issues de ces larves étaient, de la même façon, placées dans des cages avec des mâles $D_{1}$. Les deux types d'échantillons présentent une différence quant à leur signification. Dans le premier cas, on teste une grande quantité de femelles différentes provenant d'un même lieu. Dans le second cas, on étudie autant de femelles qu'il y a de pontes, car les filles qui sont étudiées présentent, dans tous les cas, le même type de croisement que leur mère.

Le tableau 1 montre les résultats de 25 tests. Le nombre de femelles et de mâles (colonne 3) varie quelque peu d'un croisement à l'autre, ceci dépendant du matériel disponible. Les proportions de femelles et de mâles sont également différentes, allant de $1 / 1$ à $4 / 1$. Néanmoins les résultats sont uniformes en ce qui concerne l'incompatibilité. Les pontes non fertiles sont rares et on les néglige, les considérant comme étant sans intérêt. Les pontes sont relevées et comptées 48 heures après le dépôt des œufs. Le tableau donne le nombre total d'œufs comptés dans chaque test (colonne 6), le nombre et le pourcentage de larves écloses (colonne 7), le nombre et le pourcentage đ'œufs embryonnés (colonne 8 ) et non embryonnés (colonne 9). A cause du manque de temps, on ne peut compter exactement toutes les pontes déposées dans un test. Les pontes en surplus ne sont mises en évidence que par l'éclosion des larves (colonne 10). 


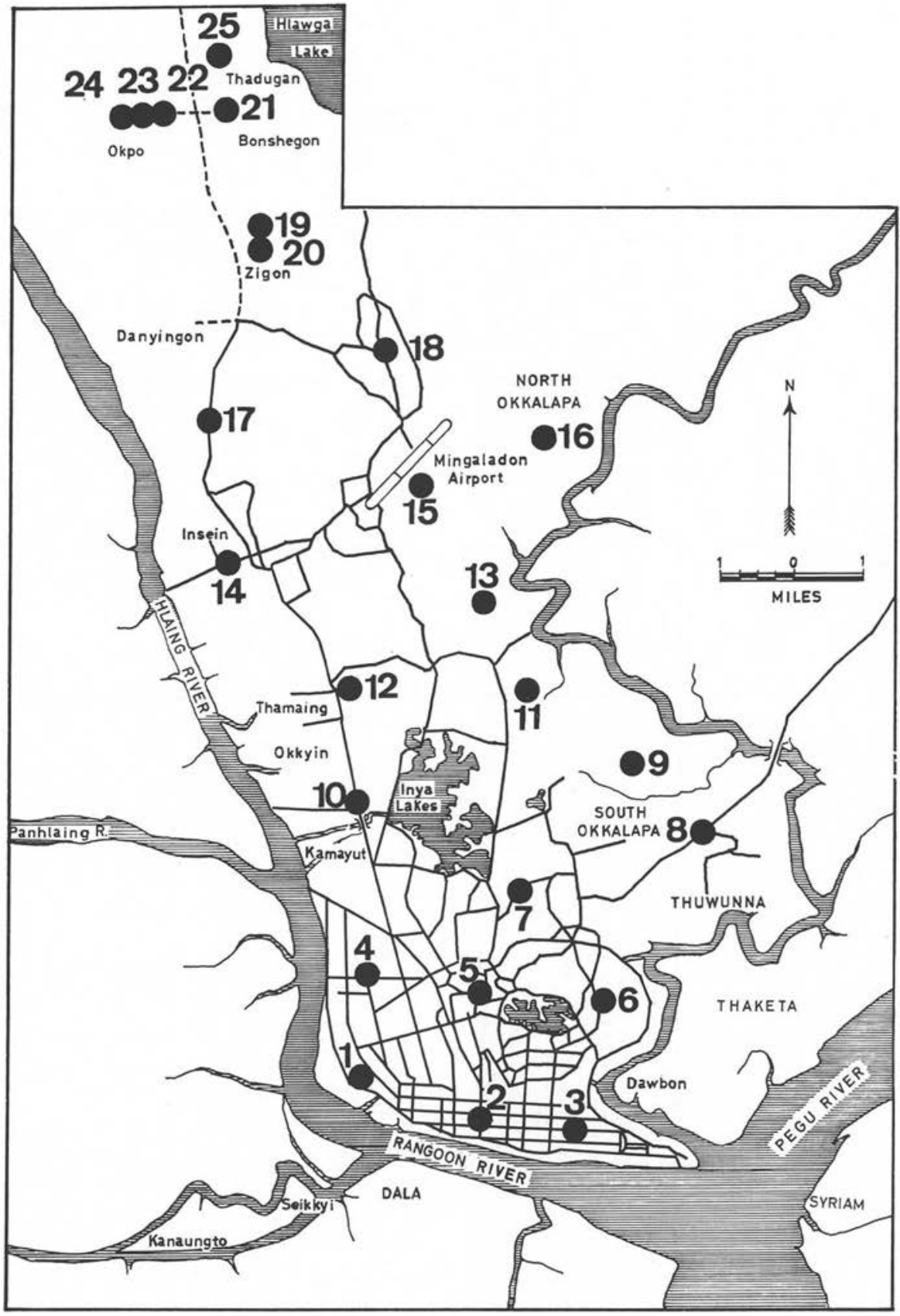

FIG. 8. - Rangoon, Birmanie, et ses environs, montrant les 25 stations de collecte d'où provenaient les insectes sauvages utilisés pour les expériences de croisement. Le tableau 1 (seconde colonne) indique les noms des stations de collecte 


\begin{tabular}{|c|c|c|c|c|c|c|c|c|c|c|c|c|}
\hline 으 & 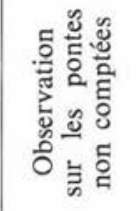 & & 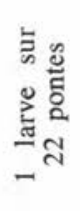 & & & 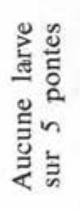 & & 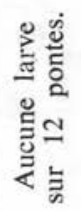 & 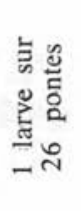 & & 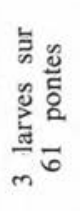 & 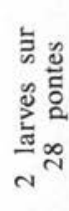 \\
\hline \multirow{2}{*}{$a$} & \multirow{2}{*}{ 尝 } & $\begin{array}{l}\text { రె } \\
\text { సi }\end{array}$ & $\begin{array}{l}\bar{N} \\
\dot{N}\end{array}$ & İ & $\begin{array}{l}\hat{b} \\
\dot{0}\end{array}$ & $\begin{array}{l}m \\
\stackrel{0}{n}\end{array}$ & aे & ๗ี & ָี & $\stackrel{0}{0}$ & $\frac{ \pm}{i}$ & $\begin{array}{l}\infty \\
\stackrel{\sim}{1}\end{array}$ \\
\hline & & $\overline{7}$ & $\bar{a}$ & $\begin{array}{l}\text { g̊ } \\
\text { vi } \\
\text { in }\end{array}$ & $\stackrel{8}{\stackrel{0}{2}}$ & है & ثั & $\frac{n}{m}$ & $\stackrel{\text { ֻ }}{\circ}$ & $\frac{n}{n}$ & ฉू & $\underset{\infty}{\infty}$ \\
\hline \multirow[t]{2}{*}{$\infty$} & \multirow{2}{*}{ | } & $=$ & $\stackrel{2}{i}$ & $\frac{n}{8}$ & ì & जิ & $\begin{array}{l}\tilde{\sigma} \\
\text { ชิ }\end{array}$ & $\begin{array}{l}\text { भे } \\
\text { से }\end{array}$ & $\begin{array}{l}\stackrel{5}{n} \\
\stackrel{5}{n}\end{array}$ & $\frac{n}{2}$ & $\begin{array}{l}+ \\
\infty \\
\infty \\
\infty\end{array}$ & $\stackrel{m}{2}$ \\
\hline & & हू & $\begin{array}{l}\bar{n} \\
\infty \\
i\end{array}$ & S̊ & $\stackrel{\infty}{+\infty}$ & 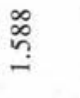 & $\bar{\swarrow}$ & $\stackrel{\infty}{\stackrel{N}{n}}$ & $\begin{array}{l}\text { స్ } \\
\text { ñ } \\
\text { ñ }\end{array}$ & $\begin{array}{l} \pm \\
\infty \\
\infty\end{array}$ & ল̊ & $\frac{7}{d}$ \\
\hline \multirow{2}{*}{$r$} & \multirow{2}{*}{ 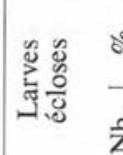 } & సু & 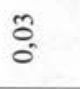 & $\stackrel{m}{0}$ & oे & $\begin{array}{l}0 \\
0\end{array}$ & 0 & oे & छे & $\stackrel{1}{0}$ & $\stackrel{\circ}{0}$ & $\theta_{0}^{\circ}$ \\
\hline & & $\nabla$ & - & 8 & $m$ & $N$ & 0 & $=$ & $N$ & N & - & $\nabla$ \\
\hline 6 & $\dot{z}: \stackrel{\mathscr{z}}{\vec{z}}$ & $\stackrel{g}{\text { g }}$ & $\begin{array}{l}\ddot{m} \\
\text { in }\end{array}$ & $\begin{array}{l}\widetilde{L} \\
\infty \\
\end{array}$ & $\begin{array}{l}\infty \\
\infty \\
\cdots \\
m\end{array}$ & $\stackrel{\text { নุ }}{\text { n் }}$ & ñ & 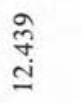 & 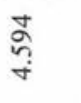 & 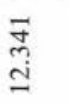 & 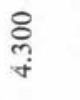 & ס্户 \\
\hline$n$ & 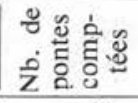 & $r$ & ి & ळి & ిల & $\approx$ & $=$ & $\approx$ & ల్ల & $\hat{b}$ & ్ల & ి \\
\hline$\forall$ & $\dot{z} \div \stackrel{\mathscr{y}}{\check{L}}$ & $r$ & กี & ळे & ర్ల & \& & $=$ & $\infty$ & ஜ & $\hat{6}$ & $\bar{\varrho}$ & $\stackrel{\infty}{n}$ \\
\hline$m$ & 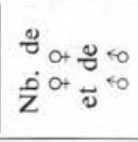 & $\begin{array}{l}\vec{y} \\
\times \\
\vec{b}\end{array}$ & $\begin{array}{l}0 \\
x \\
n\end{array}$ & $\begin{array}{l}\text { ำ } \\
\times \\
8 \\
0\end{array}$ & $\begin{array}{l}\stackrel{+}{m} \\
\times \\
\dot{m}\end{array}$ & $\begin{array}{l}\stackrel{\ominus}{e} \\
x \\
y\end{array}$ & $\begin{array}{l}0 \\
\text { in } \\
\times \\
5\end{array}$ & $\begin{array}{l}\stackrel{\infty}{+} \\
\times \\
\stackrel{+}{\simeq}\end{array}$ & $\begin{array}{l}\approx \\
\cdots \\
\approx \\
\infty\end{array}$ & $\begin{array}{l}\text { i } \\
\times \\
\text { ิ }\end{array}$ & 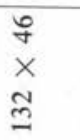 & $\begin{array}{l}\dot{y} \\
\dot{x} \\
\stackrel{a}{=}\end{array}$ \\
\hline$N$ & 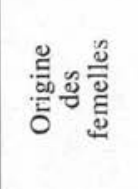 & $\frac{\dddot{0}}{\frac{0}{2}}$ & 导疍 & 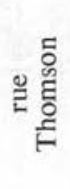 & 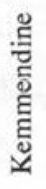 & 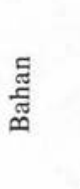 & 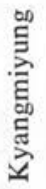 & $\begin{array}{l}\stackrel{\mathscr{E}}{\bar{\Xi}} \\
\text { ठ } \\
ن\end{array}$ & 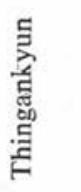 & 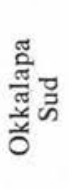 & 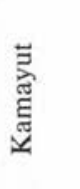 & $\underset{\nu}{\stackrel{\infty}{0}}$ \\
\hline- & $\stackrel{\circ}{Z}$ & - & $N$ & $m$ & $\forall$ & $n$ & $\bullet$ & $r$ & $\infty$ & $a$ & 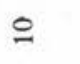 & $=$ \\
\hline
\end{tabular}




\begin{tabular}{|c|c|c|c|c|c|c|c|c|c|c|c|c|c|}
\hline 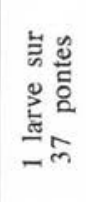 & 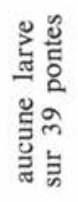 & & 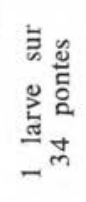 & 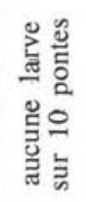 & 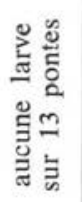 & 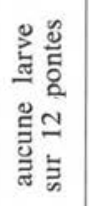 & 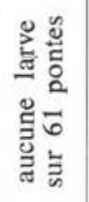 & 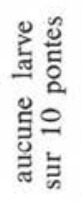 & 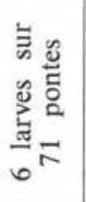 & 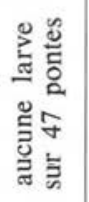 & & 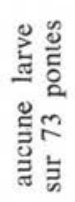 & \\
\hline$\frac{\simeq}{i n}$ & $\begin{array}{l}\tilde{n} \\
\text { ñ } \\
\tilde{n}^{2}\end{array}$ & $\frac{\Delta}{m}$ & $\begin{array}{l}\infty \\
\stackrel{0}{0} \\
\dot{\Xi}\end{array}$ & 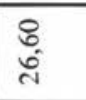 & aे & \begin{tabular}{l}
$\infty$ \\
\multirow{2}{*}{} \\
$\hat{y}$
\end{tabular} & $\begin{array}{l}\text { ñ } \\
\infty \\
\infty\end{array}$ & $\stackrel{\vec{\jmath}}{\stackrel{\sim}{\sim}}$ & $\begin{array}{l}\text { क्रे } \\
\text { है }\end{array}$ & $\begin{array}{l}\stackrel{i}{\sim} \\
\dot{\sim}\end{array}$ & $\frac{0}{9}$ & $\frac{6}{n}$ & 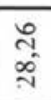 \\
\hline$\stackrel{\circ}{\stackrel{\circ}{\leftrightarrows}}$ & $\underset{+}{\stackrel{+}{+}}$ & : & ลี & $\begin{array}{l}\text { o̊ } \\
\text { in }\end{array}$ & $\stackrel{g}{\text { gे }}$ & 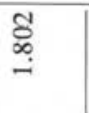 & $\tilde{n}$ & 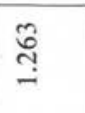 & 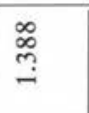 & $\underset{-}{\bar{\sigma}}$ & 离 & ֻั & $\begin{array}{l}\infty \\
\infty \\
\infty \\
0 \\
0 \\
\dot{m}\end{array}$ \\
\hline $\begin{array}{l}\infty \\
\infty \\
\text { వి }\end{array}$ & $\begin{array}{l}\infty \\
\prod_{0}^{\infty} \\
\tilde{b}\end{array}$ & $\begin{array}{l}\text { भे } \\
\text { से }\end{array}$ & $\underset{\infty}{\stackrel{d}{\sim}}$ & \begin{tabular}{l}
$\infty$ \\
\multirow{2}{*}{}
\end{tabular} & $\stackrel{n}{\approx}$ & $\frac{m}{i}$ & $\underset{\infty}{\bar{\infty}}$ & $\begin{array}{l}n \\
\hat{i}\end{array}$ & $\begin{array}{l}\text { గ్ర } \\
\text { రై }\end{array}$ & $\begin{array}{c}\text { సิ } \\
\text { ñ }\end{array}$ & 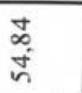 & 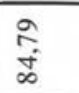 & \begin{tabular}{l}
8 \\
\hdashline \\
\end{tabular} \\
\hline 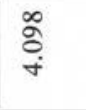 & $\stackrel{n}{\mathrm{n}}$ & $\stackrel{\infty}{\leftarrow}$ & $\stackrel{\varrho}{\dot{\sigma}}$ & $\stackrel{n}{q}$ & 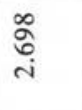 & $\frac{\tilde{\infty}}{\dot{n}}$ & $\frac{\vec{m}}{m}$ & $\underset{n}{n}$ & 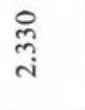 & $\frac{\tilde{\sigma}}{\dot{m}}$ & $\stackrel{\varrho}{\stackrel{g}{g}}$ & $\begin{array}{l}\overrightarrow{\text { ț }} \\
\dot{n}\end{array}$ & बे \\
\hline 0 & $\stackrel{0}{\circ}$ & $\stackrel{\text { స̆}}{0}$ & $\stackrel{\infty}{\circ}$ & I & $\stackrel{\circ}{\circ}$ & $\frac{9}{0}$ & $\stackrel{0}{0}$ & : & $\stackrel{0}{0}$ & $\ddot{0}$ & 0 & $\stackrel{n}{0}$ & $\frac{\pi}{0}$ \\
\hline 0 & $\checkmark$ & $N$ & $\theta$ & $\bar{m}$ & $\mathrm{~m}$ & 6 & - & - & $m$ & - & 0 & m & 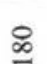 \\
\hline 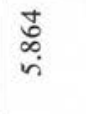 & $\hat{\kappa}$ & $\frac{\text { P }}{\sim}$ & $\frac{a}{\sigma}$ & సે & $\stackrel{\substack{n\\
}}{2}$ & ڤ్ & Ê & ڤ్రి & 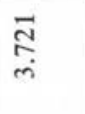 & ث્ণ & $\frac{\partial}{\dot{m}}$ & స్ర్రి & 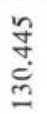 \\
\hline $\mathcal{F}$ & 요 & $n$ & ల & $\vec{q}$ & 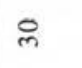 & \& & లి & 요 & 이 & 요 & ה & బ & $\underset{\infty}{\infty}$ \\
\hline 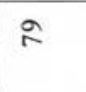 & $R$ & $n$ & t & in & $\mathscr{F}$ & $\approx$ & $\bar{a}$ & f & $\bar{\Xi}$ & $\approx$ & הี & $\cong$ & 尔 \\
\hline $\begin{array}{l}q \\
\times \\
8 \\
\stackrel{8}{0}\end{array}$ & $\begin{array}{l}\mathcal{F} \\
x \\
\stackrel{0}{\infty}\end{array}$ & $\begin{array}{l}\stackrel{0}{n} \\
\times \\
\dot{J}\end{array}$ & $\begin{array}{l}\stackrel{9}{+} \\
\times \\
a\end{array}$ & 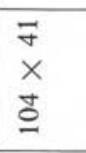 & $\begin{array}{l}m \\
x \\
\stackrel{n}{n}\end{array}$ & $\begin{array}{l}q \\
\times \\
m \\
m\end{array}$ & $\begin{array}{l}\infty \\
m \\
\times \\
\overline{0}\end{array}$ & 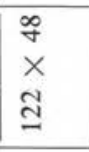 & $\begin{array}{l}\vec{J} \\
\times \\
\infty \\
\infty \\
0\end{array}$ & $\begin{array}{l}\hat{f} \\
x \\
a\end{array}$ & $\begin{array}{l}\stackrel{\infty}{+} \\
\times \\
\stackrel{0}{0} \\
\stackrel{0}{-}\end{array}$ & 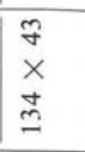 & \\
\hline 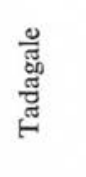 & 䓌 & 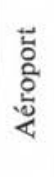 & 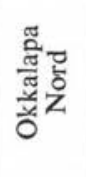 & $\begin{array}{l}\text { Iू } \\
\text { on } \\
\text { 足 }\end{array}$ & 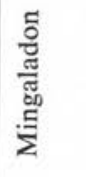 & 密芩 & 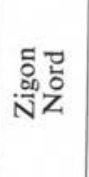 & 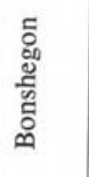 & 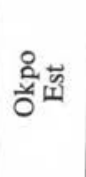 & $\begin{array}{l}\text { 总 } \\
\text { ة: }\end{array}$ & 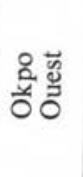 & 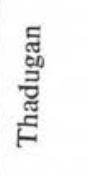 & \\
\hline 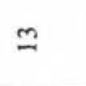 & \pm & $\cong$ & $\because$ & 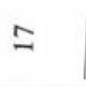 & $\stackrel{\infty}{\sim}$ & 2 & 요 & $\vec{\sim}$ & $\approx$ & $\ddot{\sim}$ & $\underset{N}{\mathbb{N}}$ & $\approx$ & స్ّ \\
\hline
\end{tabular}


Un certain nombre de larves qui éclosent (provenant aussi bien des pontes comptées que des pontes non comptées) sont conservées et élevées jusqu'à l'état adulte. Tous les imagos obtenus sont des femelles.

Tous les 25 tests présentent les caractéristiques de l'incompatibilité : fertilisation, embryogénèse, développement embryonnaire anormal, très faible pourcentage d'éclosion, tous les adultes que l'on obtient étant du sexe femelle. L'on compta en tout 130.445 œufs provenant de 880 pontes, qui ne donnèrent que 180 larves $(0,14 \%)$. Parmi les œufs restants $93.397(71,60 \%)$ étaient embryonnés et $36.858(28,26 \%)$ ne l'étaient pas. Ainsi la souche $\mathrm{D}_{1}$ est clairement incompatible, d'une manière uniforme, avec les populations de Culex, de la ville de Rangoon (tests $n^{\circ} 1-8$ ), de sa banlieue et de la zone périphérique $\left(\mathrm{n}^{\circ} 9-18\right)$ et des villages situés au Nord $\left(\mathrm{n}^{\circ} 19-25\right)$, y compris le futur village expérimental d'Okpo (n²2-24).

Une dernière expérience était encore nécessaire pour prouver l'utilité des mâles de la souche $\mathrm{D}_{1}$, à savoir, tester l'aptitude de ces mâles à entrer en compétition avec les mâles locaux. Dans ce but, on mit en place plusieurs expériences en cage, mais seule une de ces expériences sera mentionnée ici. On plaça 1.025 femelles issues de nymphes récoltées dans un gîte naturel dans une grande cage, avec 1.020 mâles de la même origine et 1.020 mâles $D_{1}$, du même âge. Les femelles, nourries ensuite sur pigeons, produisirent exactement 700 pontes. Seules 233 de ces pontes $(33,28 \%)$ éclorent normalement. Celles-ci représentent les copulations entre femelles locales et mâles correspondants. 448 pontes $(64 \%)$ ne donnèrent pas d'éclosion bien qu'embryonnées, donc, elles doivent avoir pour origine des croisements entre des femelles locales et les mâles $D_{1}$. Un petit nombre de pontes, $19(2,72 \%)$ présenta une embryogénèse partielle et une éclosion partielle. Ces pontes sont celles déposées par des femelles qui ont copulé deux fois, une fois avec un mâle local et une fois avec un mâle $\mathrm{D}_{1}$. Des expériences avec des marqueurs génétiques nous avaient appris que ceci peut être le cas dans environ $2 \%$ des copulations lorsque deux types de mâles entrent en compétition pour une femelle (Kitzmiller et Laven, 1958). A la première génération, les résultats de l'expérience de compétition montrent une nette supériorité des mâles $\mathrm{D}_{1}$. La proportion numérique de mâles $\mathrm{D}_{1}$ et de mâles locaux est égale à 1 , leur rapport d'efficacité est de $1 / 1,98$, ce qui signifie qu'ils copulent à peı près deux fois plus fréquemment que les mâles locaux. Le potentiel de reproduction de la population de la cage est réduit à 34,64 \% par les mâles $\mathrm{D}_{1}$ (Fig. 9).

En supposant que, dans les conditions normales, c'est-à-dire en l'absence de mâles $D_{1}$, la population de la cage se maintienne stable de génération en génération, en d'autres termes qu'une femelle qui a pondu produise à la génération suivante une femelle et un mâle, nous commençons une seconde génération avec 241 femelles locales et 241 mâles locaux, mais nous y ajoutons 700 mâles $\mathrm{D}_{1}$. Par l'addition de ce nombre de mâles incompatibles nous accélérons le processus dans une expérience de lâcher effectif, dans laquelle le nombre de mâles lâchés serait maintenu constant, au moins au début. La proportion numérique dans cette génération était de $1 / 2,9$. Mais une fois encore les mâles $D_{1}$ furent meilleurs que les mâles locaux, avec un taux d'efficacité de 1/4,08, car sur 149 pontes déposées, $27(18,12 \%)$ seulement éclosent, $118(79,19 \%)$ provenant 


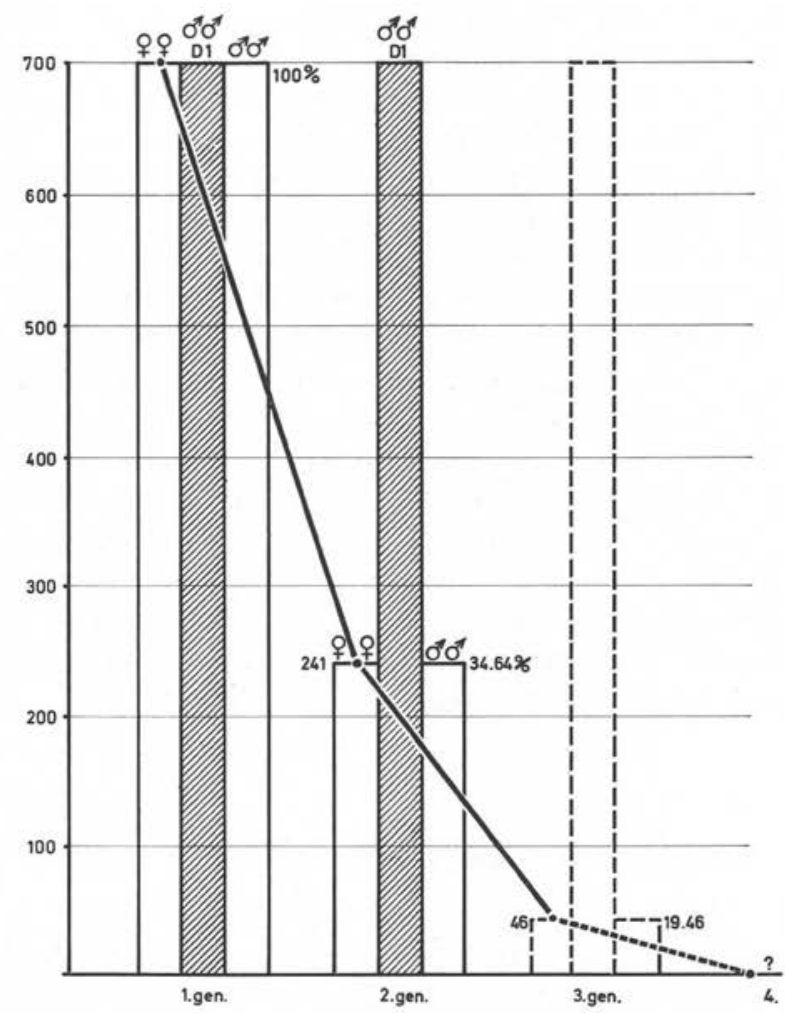

\begin{tabular}{c|c|c|c|}
\hline $\begin{array}{c}\text { ropport des deux } \\
\text { types de moles }\end{array} \quad 1: 1$ & $1: 2.9$ & $1: 15.21$ \\
\hline $\begin{array}{c}\text { efficocite des } \quad 1: 1.98 \\
\text { moles }\end{array}$ & $1: 4.08$ & \\
\hline
\end{tabular}

FIG. 9. - Résultats des expériences de compétitivité entre des mâles locaux de Culex p. fatigans de Rangoon et des mâles de la souche incompatible D1. Voir le texte pour d'autres explications

de croisements incompatibles et $4(2,69 \%)$ étant composites. La génération suivante aurait comporté, si elle s'était développée dans les mêmes conditions de population stable, comme il est mentionné ci-dessus, 46 femelles locales et 46 mâles locaux, avec encore 700 mâles $D_{1}$. Malheureusement, je n'ai pu moi-même conduire l'expérience jusqu'à la troisième génération et les résultats qui me furent rapportés ultérieurement, ne sont pas comparables aux résultats des deux premières générations. Pour cette raison je doute beaucoup que l'expérience ait été menée à bien de façon identique. Néanmoins les résultats reportés ici montrent suffisamment que les mâles de la souche $D_{1}$ peuvent rivaliser avec succès, en cage, pour la copulation avec les femelles locales. 
Nous avons encore été plus loin dans notre travail d'exploration avec les mâles $D_{1}$. Au début d'octobre 1966 , nous avions un surplus de 4.731 mâles $D_{1}$ provenant des élevages. Ils furent lâchés, pendant une période de 13 jours, en un point au centre du village d'Okpo. Déjà 4 jours après les premiers lâchers, nous trouvons 2 pontes qui n'éclosent que partiellement, première indication que l'incompatibilité agit en fait dans la nature. Un total de 239 pontes, récoltées près du point de lâcher, pendant une période de 18 jours après ce lâcher du premier groupe de mâles, comportait 11 pontes non écloses mais embryonnées et 3 pontes partiellement écloses. Il ne peut plus y avoir aucun doute sur la possibilité de lutte par utilisation de l'incompatibilité.

Je ne veux pas entrer dans les détails de nos essais en vue de développer une méthode pratique de production de masse des mâles $D_{1}$. Notre but ultime était de produire jusqu'à 5.000 mâles par jour, but que nous n'atteignîmes qu'après avoir surmonté de très nombreuses difficultés. Ces difficultés n'étaient pas du tout dues à la souche ou à l'espèce de moustique, car Culex est un des moustiques les plus faciles à élever. Les difficultés étaient avant tout liées aux techniques, au personnel et à l'organisation. Plus d'une fois le dernier essai sur le terrain était près du fiasco, à cause de phénomènes imprévus, de crédits insuffisants ou parce qu'on ne pouvait être sûr d'une partie du personnel qui aidait à la réalisation du programme.

\section{L'expérience définitive dans la nature}

Un des points les plus décisifs dans des expériences de lutte fondée sur le lâcher de mâles incompatibles ou stériles est de pouvoir obtenir une estimation digne de foi de la densité de la population, que l'on se propose de réduire. Alors seulement on peut calculer le nombre minimum de mâles nécessaires et les modalités de production de masse doivent être ajustées, conformément à ce calcul. D'importance non moins grande sont les données concernant la fluctuation d'une telle population, suivant les variations des conditions extérieures comme la température, la pluviométrie, etc... Il est évident que le lâcher de mâles, lors d'une période d'augmentation continue de la population, aura un résultat tout à fait différent du lâcher pendant une période de diminution Des données rassemblées par le «Filariasis Research Unit» de l'O.M.S. à Rangoon, mais non publiées jusqu'à maintenant, il ressort de toute évidence que, dans une certaine partie de la ville, le niveau de la population de Culex augmente de novembre à janviêr, de six fois la valeur septembre-octobre. Il y a ensuite une diminution d'environ $75 \%$ de février à septembre. Il aurait été logique de commencer les premiers lâchers immédiatement après que le plus haut niveau ait été atteint. Un tel protocole de lâchers aurait bien entendu entraîné l'obligation fâcheuse de lâcher autant de mâles qu'il y a de mâles sur le terrain, au moment de la plus haute densité de population. Néanmoins, même s'il n'est pas possible de lâcher des mâles incompatibles dès le tout début dans une proportion de $1 / 1$, toute dépression, même très faible obtenue après lâcher des mâles incompatibles accentuerait la décroissance de la population. Il n'y a pas lieu 
de supposer que les facteurs dépendant de la densité annuleraient l'effet dépresseur dû aux mâles lâchés.

En ayant à l'esprit cette idée générale, on entreprit plusieurs fois de recueillir des informations sur la densité de la population de Culex dans le village d'Okpo. La première fois en septembre et octobre 1966, mes assistants et moi-même le tentèrent, la deuxième ce fut Macdonald et ses collègues, au cours de deux semaines en janvier 1967 (Macdonald, Sebastian et Tun, 1968) et la troisième fois encore moi-même, à partir du début de février 1967, deux semaines avant les premiers lâchers.

Lors de notre première étude, nous avons utilisé trois méthodes en vue de l'estimation de la population: recensement cartographié de tous les gîtes larvaires, mesure de la productivité dans certains endroits représentatifs et extrapolation à la productivité totale journalière des adultes dans le village entier. Notre seconde tentative était de récolter les pontes jour après jour dans une certaine partie du village et d'arriver de cette façon à estimer le rôle de la population adulte dans la reproduction. Finalement, on avait combiné la récolte des œưfs dans une partie du village et les captures journalières des adultes dans les maisons de la même partie du village, ce qui donne une estimation de la population totale adulte à n'importe quel stade du cycle.

Il n'est pas possible de donner ici tous les détails de cette étude mais certaines observations sont de quelque importance pour la compréhension de l'expérience finale, spécialement les conditions très particulières du développement des larves de Culex dans le village. Nous avions très tôt remarqué qu'il n'y avait pas de larve ou très peu dans les eaux superficielles (fossés, eaux stagnantes, étangs), même pendant la saison des pluies et les deux mois qui suivent. Au moment de l'expérience de lâchers, il n'y avait pas d'eau superficielle à l'exception des étangs. Mais des sondages répétés dans les étangs ne mirent jamais en évidence de larves de Culex. Il n'y avait pas de fosses d'aisance qui sont, dans certains faubourgs de Rangoon, la source principale de Culex. La plus grande partie ou la presque totalité des gîtes larvaires du village étaient des jarres en terre cuite laissées à l'abandon, des cuvettes et des barils. On constata qu'en moyenne une habitation sur trois avait ainsi un petit gîte larvaire quelque part aux alentours (Fig. 10). Avec un total de 150 habitations cela fait à peu près 50 gîtes larvaires pour l'ensemble du village.

Des échantillons du contenu de trois types de jarres furent emportés au laboratoire et les nymphes et les larves furent élevées jusqu'à l'état adulte (Tableau 2). A partir de ces échantillons nous avons calculé que la production totale en adultes pour tout le village était d'à-peu-près 1.000 femelles et 1.000 mâles par jour.

Notre seconde méthode d'étude, à savoir la récolte journalière des pontes dans une certaine partie du village, fut utilisée en tant qu'exercice pour pouvoir apprécier ultérieurement les résultats après lâcher de mâles incompatibles. L'impact de tels mâles sur la productivité d'une population peut être estimé directement à partir des pontes qui ne sont pas écloses. La récolte des pontes avait un autre but, à savoir accumuler quelques données concernant le pourcentage d'éclosion des pontes normales. Par suite du fait que quelques pontes qui sont déposées pendant la nuit, sont perdues à cause de l'intervention des gens du village, nos valeurs des récoltes journalières de 


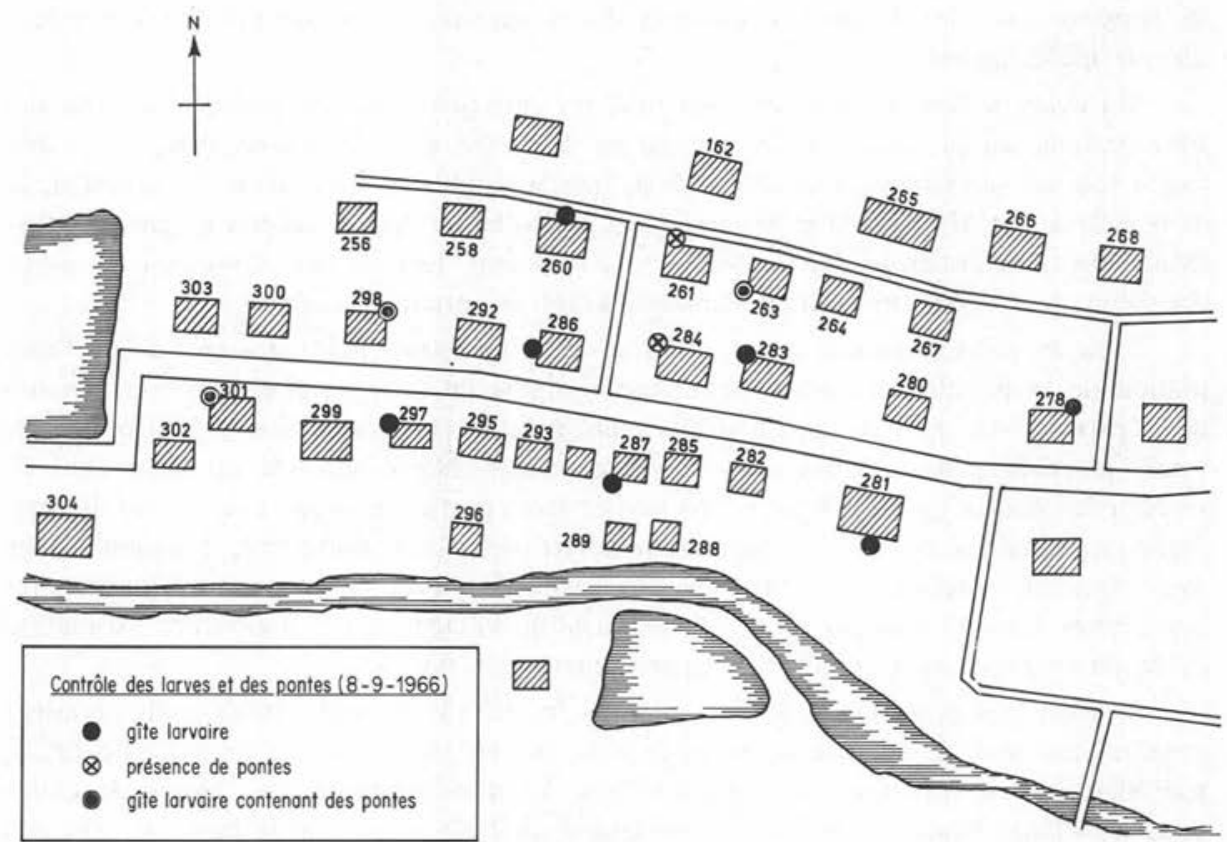

FIG. 10. - Résultats d'une étude sur les gîtes larvaires et les pontes d'une journée (8-9-1966)

Tableau 2. - Productivité dans des gîtes larvaires sélectionnés

\begin{tabular}{|c|c|c|c|c|c|}
\hline $\begin{array}{c}\text { Echantil- } \\
\text { lon } \\
n^{\circ}\end{array}$ & $\begin{array}{l}\text { Origine } \\
\text { Maison } \\
\mathrm{n}^{\circ}\end{array}$ & $\begin{array}{l}\text { Type de } \\
\text { gîte larvaire }\end{array}$ & $\begin{array}{l}\text { Partie des } \\
\text { échantillons } \\
\text { de gítes }\end{array}$ & $\begin{array}{c}\text { Productivité } \\
\text { des échantillons }\end{array}$ & $\begin{array}{c}\text { Délai } \\
\text { d'éclosion }\end{array}$ \\
\hline 1 & 241 & Jarre moyenne & $1 / 4$ & 22 ㅎํ, 19 우우 & 12 jours \\
\hline 2 & 242 & Petit pot & $1 / 4$ & $16 \hat{\delta} \hat{\sigma}, 32$ 우우 & 8 jours \\
\hline 3 & 301 & Grosse jarre & $1 / 4$ & 45 ชิ $\hat{\sigma}, 64$ 우 & 6 jours \\
\hline
\end{tabular}

pontes doivent être considérées comme minimales (Tableau 3). Nous avons récolté en moyenne un peu moins de 2 pontes par habitation et par jour. Ce qui ferait à-peuprès, 300 pontes par jour pour tout le village. Une telle valeur est très comparable à celle de la production journalière de 1.000 femelles, si nous supposons une mortalité journalière d'environ $20 \%$ et un intervalle de 4-5 jours entre l'éclosion et le dépôt de la première ponte.

Les captures dans les habitations qui constituaient la troisième méthode de notre étude furent effectuées dans quelques maisons entre le 29 septembre et le 19 octobre (Tableau 4) ; un effectif de 291 femelles fut capturé dans sept habitations. Cela fait une 
moyenne de 3,38 femelles par habitation et par jour. D'une autre récolte de femelles capturées pendant la même période, 324 sur 378 (ou 85,72 \%) déposèrent une ponte. Si on applique ce pourcentage dans le cas des 291 femelles capturées dans sept habitations, on arrive à la conclusion, par le calcul, que moins de trois femelles auraient déposé une ponte par nuit et par habitation, ce qui de nouveau correspond bien à notre valeur de deux pontes par habitation et par jour.

Tableau 3. - Résultat des collectes quotidiennes d'œufs

\begin{tabular}{|c|c|c|}
\hline Maison $\mathrm{n}^{\circ}$ & Date & $\begin{array}{l}\text { Nombre } \\
\text { de pontes }\end{array}$ \\
\hline $279,314,308,309 \ldots \ldots \ldots \ldots \ldots \ldots$ & 28- 9 & 9 \\
\hline $\begin{array}{l}279,275,273,314,311,307,309,308,310 \\
315,316,319,318 \ldots \ldots \ldots \ldots \ldots \ldots \ldots\end{array}$ & 29- 9 & 23 \\
\hline $\begin{array}{l}279,273,314,312,309,315,318,210,224 \\
225,231, \ldots \ldots \cdots \cdots \cdots \cdots \cdots \cdots \cdots \cdots \cdots \cdots\end{array}$ & 30- 9 & 20 \\
\hline $279,275,312,311,212,228,238,253 \ldots$ & $1-10$ & 13 \\
\hline $\begin{array}{l}273,309,310,318,209,210,222,227,229, \\
231,235,238,242,272 \ldots \ldots \ldots \ldots \ldots \ldots\end{array}$ & $2-10$ & 31 \\
\hline 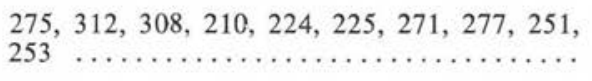 & $3-10$ & 13 \\
\hline $239,222,227,238,271,251 \ldots \ldots \ldots \ldots$ & $4-10$ & 8 \\
\hline $314,239,241,242 \ldots \ldots \ldots \ldots \ldots \ldots \ldots \ldots$ & $5-10$ & 7 \\
\hline $307,231,236,239,241,235,236,242 \ldots$ & $6-10$ & 11 \\
\hline
\end{tabular}

En rassemblant toutes ces informations récoltées dans le village en septembre et octobre 1966, nous arrivons à une estimation de la population totale d'environ 5.000 femelles et 5.000 mâles chaque jour. Par comparaison avec les études ultérieures effectuées au mois de janvier suivant, et de février à mai, on pourrait penser que ces valeurs correspondent à la période immédiatement consécutive à la saison des pluies. Mais comme nous le verrons bientôt, il peut se superposer à la fluctuation saisonnière une variation profonde dans la densité de la population, due à une intervention humaine d'un type particulier au niveau des gîtes larvaires.

Comme cela a été déjà mentionné, Macdonald et ses collègues menèrent également une étude dans le village d'Okpo en vue d'arriver à une estimation de la densité de la population. Ils capturèrent des Culex pendant une période s'étalant du 2 au 17 janvier, les marquèrent avec de la peinture et les relâchèrent dans les mêmes habitations, où ils avaient été capturés. 303 mâles et femelles furent ainsi capturés et relâchés, ce qui fait une moyenne d'environ 25 animaux par jour de capture. 15 moustiques furent recapturés une fois; un le fut deux fois. Une méthode de calcul de la taille de la population au moment de l'expérience de capture-marquage-recapture donna une esti- 
mation d'un peu plus de 2.000 animaux de chaque sexe, une autre méthode de moins de 1.000. L'effectif le plus probable de Culex était estimé à moins de 1.500 animaux de chaque sexe par jour.

Tableau 4. - Résultat des captures dans les maisons (seules les Culex femelles sont indiquées)

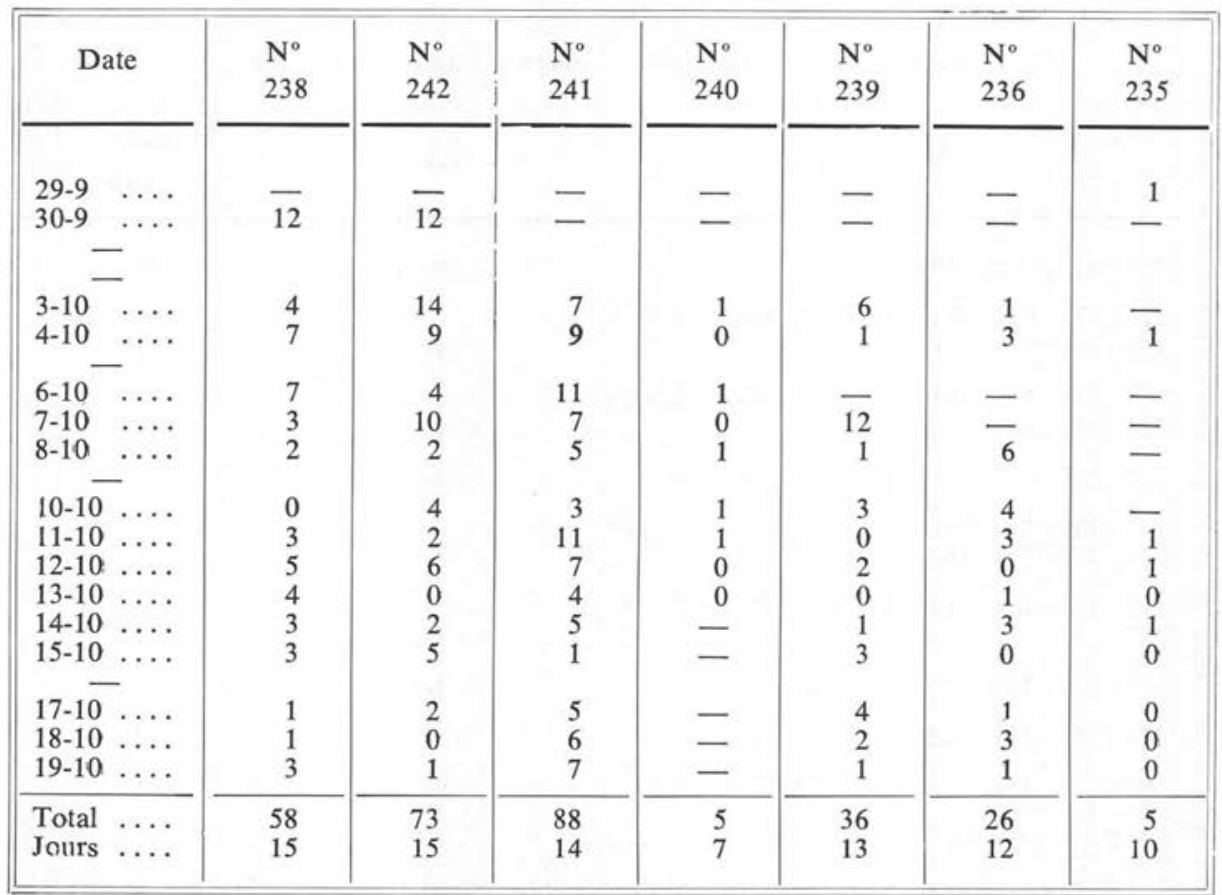

Juste deux semaines plus tard, le 2 février 1967, nous avons commencé, pour notre dernière étude, à utiliser simultanément la méthode des captures dans les habitations et celle de la récolte des pontes. Capturant dans les mêmes maisons que Macdonald et ses collègues, nous y avons trouvé des nombres quelque peu supérieurs de Culex au repos. Nous sommes arrivés à la conclusion que la population a augmenté pendant ces deux semaines d'un quart environ, ce qui a donné au total une population d'environ 2.000 animaux de chaque sexe par jour.

Très rapidement, nous avons commencé la récolte des pontes dans l'ensemble du village et ce, chaque jour (Fig. 11). Nous avons, très probablement, laissé passer quelques pontes lors des deux ou trois premières semaines de l'étude. C'est pourquoi, les valeurs initiales sont certainement trop faibles. Mais, très tôt, nous avons pu porter notre efficacité à une valeur de $99 \%$ et plus. Le village avait été séparé en neuf sections de superficie plus ou moins égale. Chacun des quatre assistants que j'avais pour m'aider parcourait chaque jour les deux sections qui lui étaient assignées, moi-même, je par- 
courais la $9^{\circ}$ section. Tous les récipients contenant de l'eau étaient inspectés et les pontes récoltées, toutes les pontes provenant de l'entourage d'une habitation étant placées dans un récipient distinct. Lorsque le temps le permettait, tous ensemble, nous parcourions de nouveau une des sections après que l'homme qui en avait la charge ait déjà récolté les œufs. Chaque fois, c'était une section différente, dont l'identité n'était connue de personne avant que nous la parcourions. Ainsi, j'avais un moyen de contrôler l'efficacité des hommes. Je suis heureux de dire que tous avaient atteint une efficacité de plus de $99 \%$, c'est-à-dire qu'ils laissaient passer en moyenne 1 ponte sur 200.

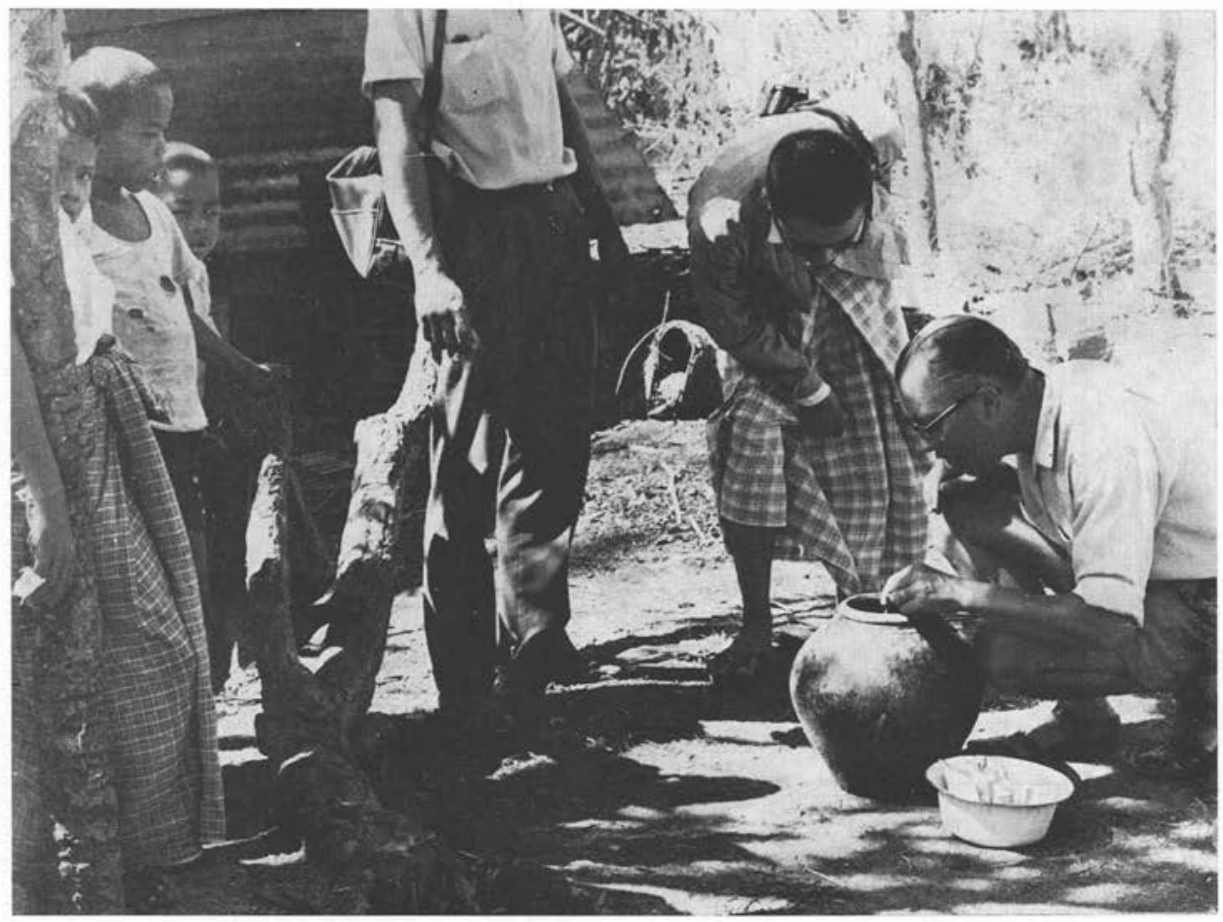

Fig. 11. - Collecte de pontes provenant d'une jarre avec présence de larves

De cette façon, on détermina chaque jour, du 16 février au $1^{\text {er }}$ mai, le nombre et la distribution des pontes pour l'ensemble du village. Les pontes étaient emportées à notre station de terrain, relevées et placées chacune dans un récipient particulier. Dans les conditions déterminantes de température, toutes les pontes normales étaient écloses 36 heures après la récolte. De cette façon, on pouvait voir aisément si une ponte provenait d'un croisement normal ou d'un croisement incompatible. Toutes les larves qui étaient écloses étaient reportées à l'endioit où les pontes correspondantes avaient été récoltées. Notre opération de récolte des œufs n'avait donc pas d'influence sur la production de moustiques. 
Revenons aux premières semaines de nos récoltes d'œufs: nous avons trouvé une moyenne de 120 pontes pour la première semaine de février, correspondant à une population d'environ 2.000 femelles par jour ; durant la deuxième semaine la valeur moyenne tombait à 82 et la troisième semaine à 70 , ce qui indiquait une légère réduction de la population qui passait peut-être à 1.500 femelles ou un peu moins.

Lors des quatre semaines suivantes, nous avons noté une très forte augmentation de la production de Culex dans le village provenant d'une multiplication par dix du nombre journalier de pontes (Fig. 12). La courbe n'est pas donnée pour les deux pre-

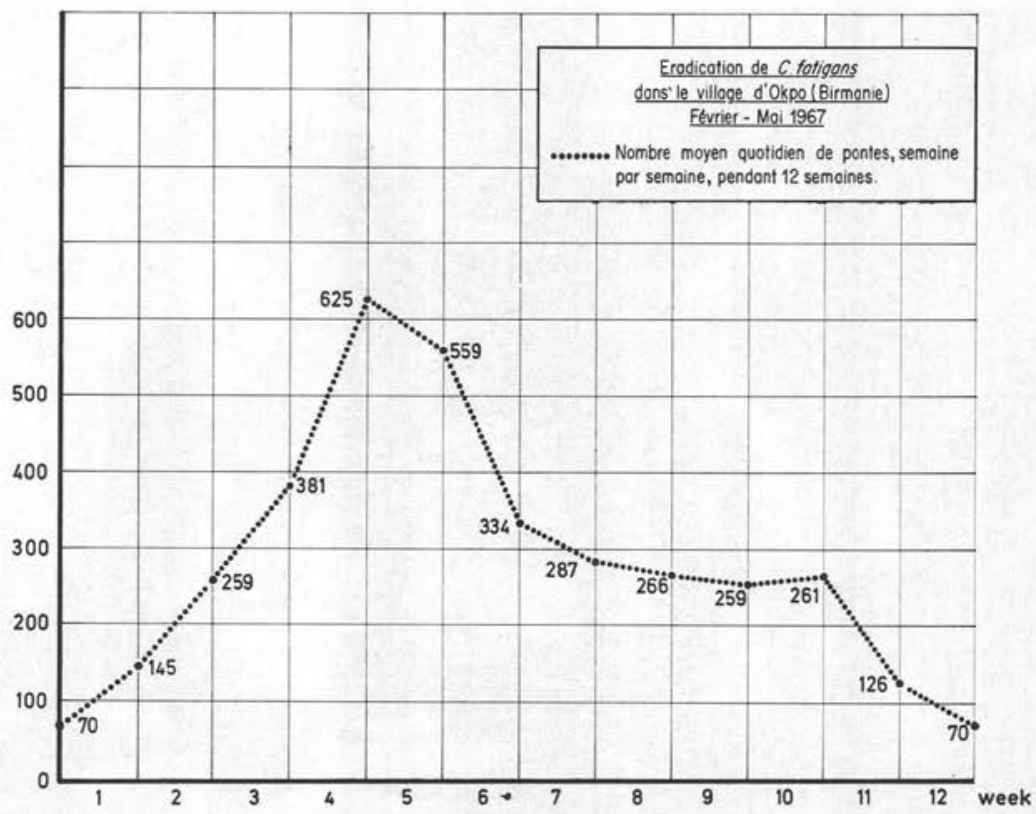

Fig. 12. - Nombre quotidien moyen de pontes de Culex p. fatigans récoltées dans le village d'Opko après lâcher de mâles incompatibles (comparer avec le tableau 5)

mières semaines de notre étude, elle débute ici avec la première semaine de lâchers, entrepris le 16 février. Très rapidement, nous trouvâmes la raison de cette augmentation. Les gens du village faisaient quelques tissages pour l'autorité locale. A peu près tous les deux mois, ils recevaient un nouveau chargement de fil de coton brut, qui doit tout d'abord être trempé dans l'eau pendant environ deux semaines, avant qu'on puisse le teindre et l'utiliser pour le tissage. En conséquence, un grand nombre de jarres contenaient du fil et de l'eau. Très rapidement, quelquefois, 24 heures après, les premières pontes de Culex apparaissaient sur l'eau de ces jarres ouvertes et le nombre de pontes augmentait de jour en jour. Très vite, ces jarres devenaient des gîtes larvaires prolifiques. C'est la raison de la forte augmentation au cours de la seconde moitié de 
février et au début de mars. Il y a un indice d'une seconde augmentation au cours de la deuxième moitié d'avril, encore due à un chargement de fil de coton, mais qui fut interrompue cette fois par nos lâchers de mâles incompatibles.

L'eau des jarres contenant du coton frais doit exercer une attraction spéciale pour les femelles en quête d'un endroit pour déposer leurs œufs. Mais, contrairement à n'importe quel autre type d'eau très attractive, l'eau contenant du fil de coton représente également un très bon milieu pour ces larves. L'autre type de gîte attirant beaucoup de femelles correspondait à l'eau de lavage du riz. D'habitude, cette eau était donnée au bétail, buffles ou cochons, mais elle stagnait en maints endroits, et attirait les femelles en grand nombre. Cependant, les larves succombaient généralement dans ce type d'eau, très probablement à cause de la forte acidité qui s'y manifestait.

Chaque jour, l'accroissement du nombre de pontes pendant les mois de février et de mars correspond à un accroissement de la densité de la population d'environ 1.500 à la mi-février à 10.000 et peut-être presque 15.000 animaux des deux sexes à la mimars. Ceci correspond bien entendu à une population encore très faible en comparaison de la population totale de Culex existant sur un seul mille carré, à Rangoon, estimée à 40.000.000 (Knipling et al., 1968, Annexe).

Comme nous arrivons finalement à l'expérience de lâchers, je voudrais dès le début bien préciser le fait qu'elle n'était destinée qu'à prouver que les mâles incompatibles pouvaient rivaliser dans la nature avec les mâles locaux et qu'ils pouvaient exercer une influence sur une population naturelle en diminuant la productivité et donc la densité de la population. Notre espoir était évidemment de parvenir à l'éradication totale des moustiques dans le village.

Il y eut beaucoup de difficultés à surmonter, avant d'effectuer des lâchers suffisants et efficaces. La première de toutes fut que le délai fixé et imparti pour la production en masse de mâles incompatibles ne fut pas respecté par les personnes responsables de cette partie du travail. On m'avait prévenu que, dès le jour de mon arrivée à Rangoon, c'est-à-dire dès le $1^{\text {er }}$ février, 4 à 5.000 mâles incompatibles seraient disponibles en moyenne chaque jour. Ce que je trouvais, c'était un petit élevage, donnant péniblement 50 mâles et femelles par jour. Pas de mâles pour les lâchers, rien de préparé pour une production plus importante, pas même les bacs et les autres installations nécessaires à une production de masse.

Je ne voudrais pas détailler les efforts pratiquement désespérés que j’ai déployés pour obtenir de l'aide, du matériel, des moyens de transport et autres pendant les toutes premières semaines. Finalement, le 16 février, nous avions quelques centaines de mâles en vue du lâcher (Tableau 5). Pendant les quatre semaines suivantes, nous n'avions qu'une moyenne de 1.350 mâles par jour à lâcher. Il fallait comparer ce nombre au nombre de mâles présents pendant cette période. Comme il a été noté auparavant, le nombre de mâles dans le village était passé d'à-peu-près 1.500 par jour à environ 10.000 pendant ces quatre premières semaines. Comme les droites obliques l'indiquent sur le graphique (Fig. 13), cette partie de l'expérience devrait, en fait, ne pas être prise en considération. Ce n'était qu'un départ prématuré avec des moyens nettement insuffisants. Néanmoins, avec un taux probable d'un mâle incompatible pour un nombre de 
Tableau 5. - Observations quotidiennes durant l'expérience de lâcher dans le village d'Okpo: nombre de mâles de la souche $\mathrm{D}_{1}$ relâchés, nombre de pontes récoltées, pourcentage de pontes écloses et de pontes non écloses

\begin{tabular}{|c|c|c|c|c|c|}
\hline $\begin{array}{l}\text { Date } \\
1967\end{array}$ & 部 & $\begin{array}{l}\mathrm{Nb} \text {. } \\
\text { de mâles } \\
\text { relâchés }\end{array}$ & $\begin{array}{l}\mathrm{Nb} . \\
\text { de pontes } \\
\text { récoltées }\end{array}$ & $\begin{array}{c}\% \\
\text { de pontes } \\
\text { écloses }\end{array}$ & $\begin{array}{c}\% \\
\text { de pontes } \\
\text { non écloses }\end{array}$ \\
\hline $\begin{array}{ll}16-2 & \ldots \\
17-2 & \ldots \\
18-2 & \ldots \\
19-2 & \ldots \\
20-2 & \ldots\end{array}$ & & $\begin{array}{r}1.000 \\
2.000 \\
100 \\
500\end{array}$ & $\begin{array}{l}53 \\
73 \\
87 \\
65 \\
84\end{array}$ & $\begin{array}{l}100 \\
100 \\
100 \\
100 \\
100\end{array}$ & $\begin{array}{l}z \\
z \\
-\end{array}$ \\
\hline $\begin{array}{ll}21-2 & \ldots \\
22-2 & \ldots \\
23-2 & \ldots \\
24-2 & \ldots \\
25-2 & \ldots \\
26-2 & \ldots \\
27-2 & \ldots\end{array}$ & 1 & $\begin{array}{r}500 \\
900 \\
1.200 \\
800 \\
1.000 \\
1.000 \\
500\end{array}$ & $\begin{array}{r}87 \\
103 \\
135 \\
274 \\
161 \\
96 \\
169\end{array}$ & $\begin{array}{c}100 \\
98,3 \\
96,3 \\
93,1 \\
94,5 \\
93,7 \\
93,5\end{array}$ & $\begin{array}{l}\overline{1,7} \\
3,7 \\
6,9 \\
5,5 \\
6,3 \\
6,5\end{array}$ \\
\hline $\begin{aligned} 28-2 & \ldots \\
1-3 & \ldots \\
2-3 & \ldots \\
3-3 & \ldots \\
4-3 & \ldots \\
5-3 & \ldots \\
6-3 & \ldots\end{aligned}$ & 2 & $\begin{array}{r}1.000 \\
1.000 \\
800 \\
1.200 \\
1.500 \\
2.000 \\
2.500\end{array}$ & $\begin{array}{l}329 \\
312 \\
265 \\
283 \\
217 \\
310 \\
197\end{array}$ & $\begin{array}{l}94,0 \\
94,3 \\
94,4 \\
95,2 \\
95,4 \\
97,1 \\
91,9\end{array}$ & $\begin{array}{l}6,0 \\
5,7 \\
5,6 \\
4,8 \\
4,6 \\
2,9 \\
9,1\end{array}$ \\
\hline $\begin{aligned} 7-3 & \ldots \\
8-3 & \ldots \\
9-3 & \ldots \\
10-3 & \ldots \\
11-3 & \ldots \\
12-3 & \ldots \\
13-3 & \ldots\end{aligned}$ & 3 & $\begin{array}{l}2.000 \\
2.000 \\
2.300 \\
2.500 \\
2.200 \\
2.000 \\
2.000\end{array}$ & $\begin{array}{l}262 \\
288 \\
248 \\
257 \\
380 \\
628 \\
585\end{array}$ & $\begin{array}{l}89,4 \\
91,4 \\
86,3 \\
87,6 \\
86,4 \\
90,0 \\
87,5\end{array}$ & $\begin{array}{r}10,6 \\
8,6 \\
13,7 \\
12,4 \\
13,6 \\
10,0 \\
12,5\end{array}$ \\
\hline $\begin{array}{ll}14-3 & \ldots \\
15-3 & \ldots \\
16-3 & \ldots \\
17-3 & \ldots \\
18-3 & \ldots \\
19-3 & \ldots \\
20-3 & \ldots\end{array}$ & 4 & $\begin{array}{l}2.000 \\
3.000 \\
5.000 \\
5.000 \\
5.000 \\
5.000 \\
5.000\end{array}$ & $\begin{array}{l}639 \\
569 \\
631 \\
762 \\
586 \\
558 \\
629\end{array}$ & $\begin{array}{l}86,8 \\
90,6 \\
89,1 \\
89,7 \\
85,9 \\
87,5 \\
88,9\end{array}$ & $\begin{array}{r}13,2 \\
9,4 \\
10,9 \\
10,3 \\
14,1 \\
12,5 \\
11,1\end{array}$ \\
\hline $\begin{array}{ll}21-3 & \ldots \\
22-3 & \ldots \\
23-3 & \ldots \\
24-3 & \ldots \\
25-3 & \ldots \\
26-3 & \ldots \\
27-3 & \ldots\end{array}$ & 5 & $\begin{array}{l}5.000 \\
5.000 \\
5.000 \\
5.000 \\
5.000 \\
5.000 \\
5.000\end{array}$ & $\begin{array}{l}696 \\
607 \\
652 \\
634 \\
544 \\
345 \\
433\end{array}$ & $\begin{array}{l}82,0 \\
81,7 \\
82,4 \\
80,6 \\
80,7 \\
79,8 \\
76,5\end{array}$ & $\begin{array}{l}18,0 \\
18,3 \\
17,6 \\
19,4 \\
19,3 \\
20,2 \\
23,5\end{array}$ \\
\hline $28-3 \ldots$ & & 5.000 & 401 & 76,9 & 23,1 \\
\hline
\end{tabular}




\begin{tabular}{|c|c|c|c|c|c|}
\hline $\begin{array}{l}\text { Date } \\
1967\end{array}$ & .气 & $\begin{array}{l}\text { Nb. } \\
\text { de mâles } \\
\text { relâchés }\end{array}$ & $\begin{array}{l}\mathrm{Nb} . \\
\text { de pontes } \\
\text { récoltées }\end{array}$ & $\begin{array}{c}\% \\
\text { de pontes } \\
\text { écloses }\end{array}$ & $\begin{array}{c}\% \\
\text { de pontes } \\
\text { non écloses }\end{array}$ \\
\hline $\begin{aligned} 29-3 & \ldots \\
30-3 & \ldots \\
31-3 & \ldots \\
1-4 & \ldots \\
2-4 & \ldots \\
3-4 & \ldots\end{aligned}$ & 6 & $\begin{array}{l}5.000 \\
5.000 \\
5.000 \\
4.000 \\
2.250 \\
3.000\end{array}$ & $\begin{array}{l}332 \\
316 \\
284 \\
359 \\
373 \\
286\end{array}$ & $\begin{array}{l}79,4 \\
78,5 \\
75,1 \\
73,0 \\
71,1 \\
72,1\end{array}$ & $\begin{array}{l}20,6 \\
21,5 \\
24,9 \\
27,0 \\
28,9 \\
27,9\end{array}$ \\
\hline $\begin{aligned} 4-4 & \ldots \\
5-4 & \ldots \\
6-4 & \ldots \\
7-4 & \ldots \\
8-4 & \ldots \\
9-4 & \ldots \\
10-4 & \ldots\end{aligned}$ & 7 & $\begin{array}{l}5.000 \\
5.000 \\
2.500 \\
2.500 \\
5.000 \\
5.000 \\
5.000\end{array}$ & $\begin{array}{l}313 \\
348 \\
272 \\
205 \\
302 \\
277 \\
290\end{array}$ & $\begin{array}{l}67,4 \\
76,5 \\
65,5 \\
69,8 \\
68,3 \\
70,1 \\
68,7\end{array}$ & $\begin{array}{l}32,6 \\
23,5 \\
34,5 \\
30,2 \\
31,7 \\
29,9 \\
31,3\end{array}$ \\
\hline $\begin{array}{ll}11-4 & \ldots \\
12-4 & \ldots \\
13-4 & \ldots \\
14-4 & \ldots \\
15-4 & \ldots \\
16-4 & \ldots \\
17-4 & \ldots\end{array}$ & 8 & $\begin{array}{l}5.000 \\
5.000 \\
5.000 \\
5.000 \\
5.000 \\
5.000 \\
5.000\end{array}$ & $\begin{array}{l}321 \\
371 \\
289 \\
252 \\
262 \\
196 \\
170\end{array}$ & $\begin{array}{l}69,8 \\
63,1 \\
60,9 \\
59,6 \\
60,0 \\
59,2 \\
54,2\end{array}$ & $\begin{array}{l}30,2 \\
36,9 \\
39,1 \\
40,4 \\
40,0 \\
40,8 \\
45,8\end{array}$ \\
\hline $\begin{array}{ll}18-4 & \cdots \\
19-4 & \ldots \\
20-4 & \ldots \\
21-4 & \ldots \\
22-4 & \cdots \\
23-4 & \cdots \\
24-4 & \cdots\end{array}$ & 9 & $\begin{array}{l}5.000 \\
5.000 \\
5.000 \\
5.000 \\
5.000 \\
5.000 \\
5.000\end{array}$ & $\begin{array}{r}223 \\
208 \\
136 \\
257 \\
331 \\
289 \\
371\end{array}$ & $\begin{array}{l}50,7 \\
54,9 \\
47,8 \\
48,7 \\
44,2 \\
46,8 \\
41,6\end{array}$ & $\begin{array}{l}49,3 \\
54,1 \\
52,2 \\
51,3 \\
55,8 \\
53,2 \\
58,4\end{array}$ \\
\hline $\begin{aligned} 25-4 & \ldots \\
26-4 & \ldots \\
27-4 & \ldots \\
28-4 & \ldots \\
29-4 & \ldots \\
30-4 & \ldots \\
1-5 & \ldots\end{aligned}$ & 10 & $\begin{array}{r}5.000 \\
2.500 \\
2.500 \\
2.000 \\
600 \\
5.000 \\
5.000\end{array}$ & $\begin{array}{l}327 \\
305 \\
285 \\
270 \\
264 \\
290 \\
231\end{array}$ & $\begin{array}{l}36,1 \\
33,5 \\
30,7 \\
27,5 \\
30,0 \\
26,3 \\
22,6\end{array}$ & $\begin{array}{l}63,9 \\
66,5 \\
69,3 \\
72,5 \\
70,0 \\
73,7 \\
77,4\end{array}$ \\
\hline $\begin{array}{ll}2-5 & \ldots \\
3-5 & \cdots \\
4-5 & \cdots \\
5-5 & \cdots \\
6-5 & \cdots \\
7-5 & \cdots \\
8-5 & \cdots\end{array}$ & 11 & $\begin{array}{l}5.000 \\
5.000 \\
5.000 \\
5.000 \\
5.000 \\
- \\
-\end{array}$ & $\begin{array}{r}180 \\
150 \\
149 \\
123 \\
98 \\
88 \\
91\end{array}$ & \begin{tabular}{r|r}
17,8 \\
16,6 \\
15,5 \\
11,7 \\
8,2 \\
6,9 \\
4,4
\end{tabular} & $\begin{array}{l}82,2 \\
83,3 \\
84,5 \\
88,6 \\
91,8 \\
93,1 \\
95,6\end{array}$ \\
\hline $\begin{aligned} 9-5 & \ldots \\
10-5 & \ldots\end{aligned}$ & 12 & - & $\begin{array}{l}75 \\
65\end{array}$ & 二 & $\begin{array}{l}100 \\
100\end{array}$ \\
\hline
\end{tabular}


mâles locaux allant jusqu'à 10 ou plus, on peut noter déjà un faible impact sur la population, se caractérisant à la fin des quatre premières semaines par un pourcentage de pontes non écloses de $12 \%$. Le nombre suffisant de 5.000 mâles par jour ne fut disponible pour la première fois que le 16 mars. Ce nombre put être maintenu à peu près constant tous les jours jusqu'à la fin de l'expérience. Mais on avait perdu un temps précieux, car il ne restait plus que huit semaines avant le début des pluies.

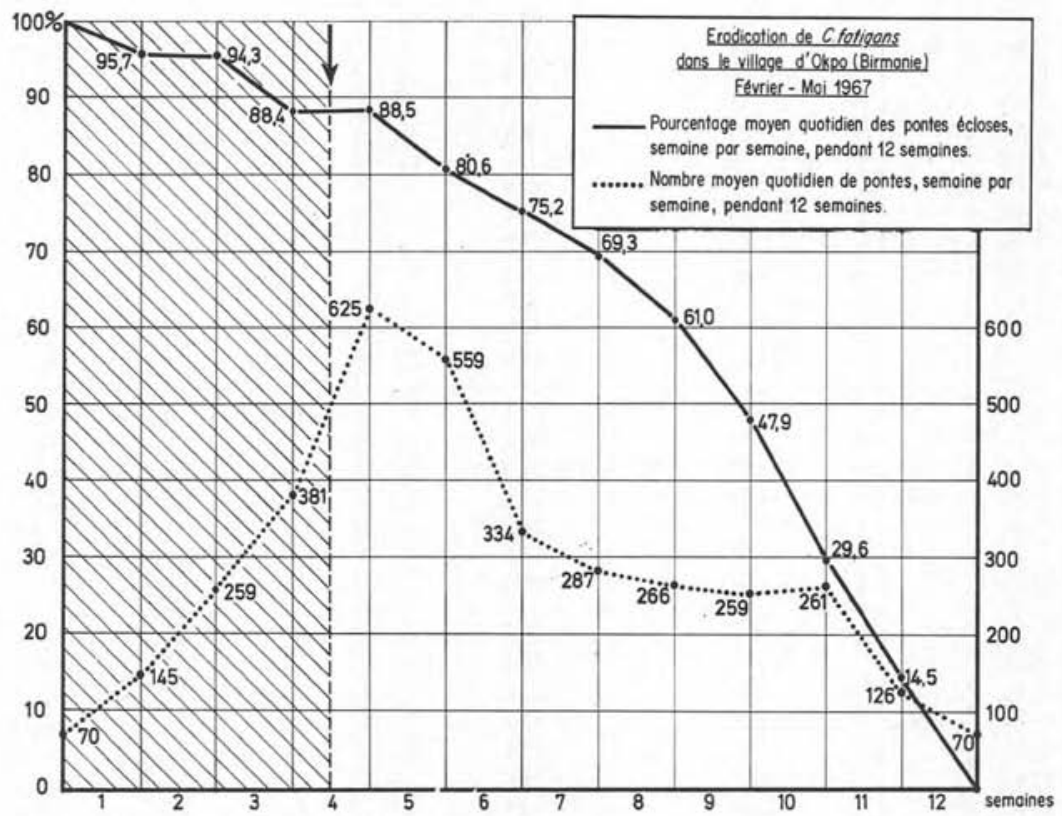

Fig. 13. - Résultats de l'expérience pour éradication de Culex fatigans dans le village d'Opko (Birmanie), février-mai 1967. Pourcentage moyen quotidien des pontes écloses, semaine par semaine, pendant 12 semaines

Il n'y a que peu de choses à dire au sujet des lâchers. Les mâles incompatibles étaient transportés au village en lots de 200 à 400 dans une petite cage. Une fois terminées les études et fait le travail préliminaire, on lâchait les mâles en 20-25 endroits plus ou moins espacés, dans tout le village, et plus particulièrement dans les jarres hébergeant les larves.

L'influence du nombre suffisant de mâles incompatibles après les quatre semaines devient immédiatement évidente. Durant ces quatre semaines, il y eut un pourcentage moyen d'augmentation de $3 \%$ des pontes incompatibles chaque semaine. Ensuite, il atteignit à peu près $8 \%$ en une semaine. Il y a un léger ralıntissement pendant les semaines 6 et 7 avec des pourcentages de 5,4 et 5,9, ceci étant probablement dû à l'augmentation antérieure de la population. Mais, à partir de la huitième semaine, le pourcentage de pontes incompatibles augmente de nouveau : 8,3 pendant la 
huitième semaine, 13,1 pendant la neuvième, 18,3 pendant la dixième, 15,1 la semaine suivante et 14,5 pendant la douzième semaine. La situation à la fin de l'expérience était la suivante : pendant la douzième semaine, il $\mathrm{y}$ avait encore une production moyenne de 70 pontes par jour, mais avec un pourcentage d'éclosion diminuant jusqu'à atteindre l'arrêt total de la productivité, avec $100 \%$ de pontes non viables, pendant les deux derniers jours où le travail dans le village était encore possible. Malheureusement, la mousson débuta le 11 mai et l'expérience a dû être arrêtée.

Il est certain que des larves et des adultes étaient encore vivants dans le village. Une petite fraction des femelles (peut-être 4-5\%, c'est-à-dire deux ou trois femelles) aurait déposé une ponte viable la semaine suivante. Tous les mâles issus des que!ques larves encore existantes auraient trouvé une population de mâles à raison peut-être d'un mâle local pour 500 mâles incompatibles. Ainsi, je n'ai aucun doute au sujet du résultat qu'aurait donné le lâcher pendant deux semaines supplémentaires : l'éradication totale. Vous comprendrez maintenant le caractère assez rude de mes remarques concernant le retard d'à-peu-près six semaines dans les lâchers, au début de l'expérience.

Cette expérience a entraîné diverses réactions. Un critique, ancien membre de la direction de l'O.M.S., a dit ceci: «Aucune preuve d'observations applicables ultérieurement n'ayant été présentée, et des larves, des pupes, des adultes de Culex pipiens fatigans se trouvant encore dans la zone de l'expérience, au moment où l'arrivée des pluies modifiait profondément les disponibilités locales en gîtes larvaires, le vieux verdict écossais " non prouvé" s'applique donc. (Laird, 1967). La question est posée à tout le monde, d'estimer ce qui a été prouvé et ce qui ne l'a pas été. A mon sens, ce qui importe, ce n'est pas la disponibilité en gîtes larvaires, mais la production de descendance. Celle-ci avait baissé nettement et était vouéz à une interruption totale.

A l'opposé, après l'expérience de Birmanie, certains journaux titraient, avec exagération :

* The answer is in sex not powder ».

De toute manière, en répétant l'expérience selon la même méthode de répondre aux critiques. Si un tel programme est mieux organisé et si l'on dispose du temps nécessaire, il sera possible de prouver ou de réfuter ce que nous avançons, à savoir l'éradication totale.

\section{Bibliographie}

BARR (A. R.), 1966: - Cytoplasmic incompatibility as a mean of eradication of Culex pipiens L. Proc. 33. Ann. Conf. California Mosq. Control Ass., 32-35.

Kitzmiler (J. B), 1958. - X-ray induced mutations in the mosquito Culex fatigans. Exp. Parasitol., 7, 439-462.

- et LAven (H.), 1958. - Tests for multiple fertilization in Culex mosquitoes by the use of genetic markers. Amer. J. Hyg., 67, 207-213. 
Knipling (E. F.), Laven (H.), Craig (G. B.), Pal (R.), Kitzmiller (J. B.), Smith (C. N) et Brown (A.W. A), 1968. - Genetic control of insects of public health importance. Bull. Wrld. Hith. Org., 38, 421-438.

LAIRD (M.), 1967. - Eradication of Culex pipiens fatigans through cytoplasmic incompatibility. Nature, 216, 1358.

LAVEN (H.), 1953. - Reziprok unterschiedliche Kreuzbarkeit von Stechmücken (Culicidae) und ihre Deutung als plasmatische Vererbung. Z. Vererbungsl., 85, 118-136.

-, 1955. - Strahleninduzierte Mutationen in Culex pipiens. Z. f. Naturforsch., 106, 320322.

-, 1956. - X-ray induced mutations in mosquitoes. Proc. R. Ent. Soc. Lond. (A.), 31, 17-19.

—, $1957 a$. - Vererbung durch Kerngene und das Problem der außerkaryotischen Vererbung bei Culex pipiens. I. Kernvererbung. Z. Vererbungsl., 88, 443-477.

-, 1957 b. - Vererbung durch Kerngene und das Problem der außerkaryotischen Vererbung bei Culex pipiens. II. Außerkaryotische Vererbung. Z. Vererbungsl., 88, 478-516.

- 1959. - Speciation by cytoplasmic isolation in the Culex pipiens complex. Cold Spring Habor Symp. Quant. Biol., 24, 166-173.

-, 1967 a. - Formal genetics of Culex pipiens. In : Genetics of Insect Vectors of Disease, ed. J. W. Wright et R. Pal, Amsterdam (Elsevier Publ. Co.), 17-65.

-, 1967 b. - Speciation and evolution in Culex pipiens. In : Genetics of Insect Vectors of Disease, ed. J. W. Wright et R. Pal, Amsterdam (Elsevier Publ. Co.), 251-275.

-, 1967 c. - Eradication of Culex pipiens fatigans through cytoplasmic incompatibility. Nature, 216, 383-384.

-, 1969. - Incompatibility tests in the Culex pipiens complex. Part. II. Egyptian strains Mosq. News, 29, 74-83.

Macdonald (W.W.), Sebastian (A.) et Tun (M. M.), 1968. - A mark-release-recapture experiment with Culex pipiens fatigans in the village of Okpo, Burma. Ann. Trop. Med. Parasitol., 62, 200-209.

Marshall (J. F), 1938. - The British Mosquitoes, London.

McClelland (G. A. H.), 1967. - Speciation and evolution in Aedes. In : Genetics of Insect Vectors of Disease, ed. J.W. Wright et R. Pal, Amsterdam (Elsevier Publ. Co.), 277-311.

Roubaud (E.), 1941. - Phénomènes d'amixie dans les intercroisements de Culicides du groupe pipiens. C. r. Acad. Sci. (Paris), 212, 257-259. 